\title{
Commod
}

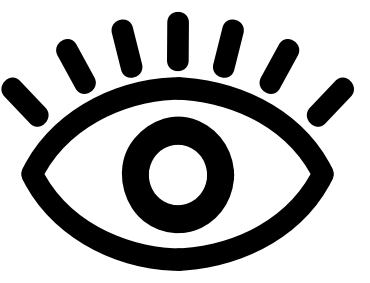

Observation manual for collective serious games

William's Daré, Emeline Hassenforder, Anne Dray For the ComMod group 



\section{Observation manual for collective serious games}

William's Daré

UPR GREEN, UMR SENS, CIRAD, Univ. Montpellier

williams.dare@cirad.fr

Emeline Hassenforder

UMR G-EAU, IRSTEA/CIRAD, Univ. Montpellier

emeline.hassenforder@cirad.fr

Anne Dray

ForDev, ETH Zurich

anne.dray@usys.ethz.ch

ComMod/CIRAD 2020 


\section{ComMod/CIRAD 2020}

ComMod Association

11 Place Adolphe Chérioux

75015 Paris

France

www.commod.org

\section{CIRAD}

Avenue Agropolis

34398 Montpellier Cedex 5

France

www.cirad.fr

\section{ISBN 978-2-87614-765-2}

Legal deposit December 2020

How to cite this document:

Daré W., Hassenforder E., Dray A., 2०२७. Observation manual for collective serious games. CIRAD, Montpellier, $68 \mathrm{p}$.

https://doi.org/10.19182/agritrop/00144

This work is made available under the terms of the

Licence Creative Commons : CC BY NC SA

You may exploit it for non-commercial purposes, always quoting its authors, and create derivative works, provided they are

distributed under a licence identical to that governing the original work.
Layout:

Ola Dolinska

ola@yare.fr

\section{(c) (i) () (2)}




\section{Special thanks}

A large part of the ComMod community has contributed to this book, either through specific comments on the document or through discussions and feedback that have contributed to our reflection. We were inspired by many games set up by trainees, $\mathrm{PhD}$ students, post-docs, researchers and practitioners.

Many thanks to them!

We especially thank Clémence Moreau, Christophe Le Page and Timothée Fouqueray for their careful rereading, as well as Juliette Cerceau and Eva Perrier for testing a preliminary version of the manual during their game sessions.











\section{Preamble}

The construction of this manual has been the result of lengthy experience of the observation ${ }^{1}$ and practice of serious games by the members of the ComMod group (www.ComMod.org).

The idea of this manual was based on three points:

1. The observation, monitoring and evaluation of serious games is often neglected, due to a lack of time, resources or consideration.

2. When observation protocols are implemented, usually it is for research purposes (for 'cold' analysis), but rarely for the 'hot' debriefing of game sessions.

3. Observation of serious games is often seen as a 'war machine', i.e. a cumbersome and tiresome device to set up.

This manual is intended to be simple and intuitive in helping to build an observation protocol adapted to the needs of the people implementing and using serious games.

1. Words in italics can be found in the glossary. 


\section{Table of contents}

Special thanks 5

Preamble 7

Table of contents 8

Illustrated table of contents 9

\section{Framing}

What serious games are we talking about? 11

In what context should this manual be used? 13

Preliminary note on the ethical and legal aspects of data collection 14

Why observe a serious game session? 15

What data are we talking about? 16

What equipment should I use to observe? 17

Who is observing? 18

What posture is appropriate for the observer? 19

When should we observe? 20

How to build the observation protocol? 21
Observation protocol

Step 1: Specify the objective(s) of the gaming session 22

Step 2: Identify the elements to be observed

according to the session's objectives 24

Step 3: Create the observation tools 31

Step 4: Collect data using the observation tools 38

Step 5: Mobilise the observed data 40

Overview of the steps to follow 48

To go further 49

Glossary 52

Bibliography 54

Appendices 57 


\section{Illustrated table of contents}

Framing p. 11-20

Why? Who? What?
OBSERVATION
When? How?

5-step Observation Protocol p. 21-47 + Overview p. 48

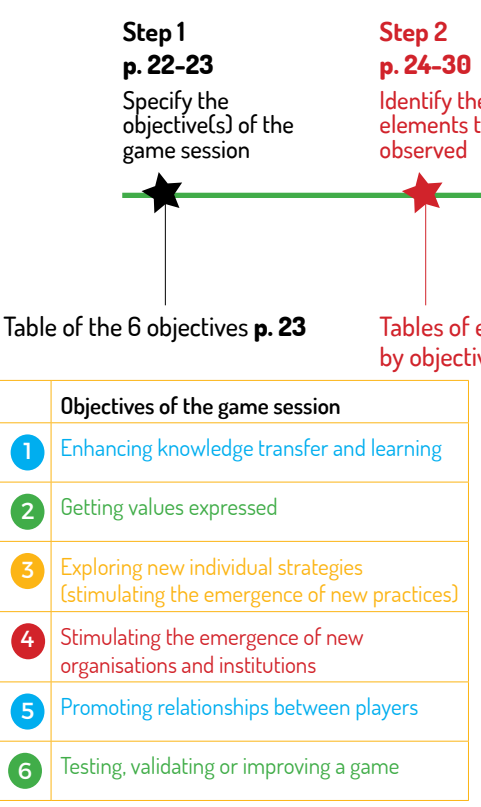

Game session description sheet Appendix 1
Step 4
p. 38-39
Collect data using
p. 40-47
observation tools
Mobilise the
p. 31-37

Create

observation

tools
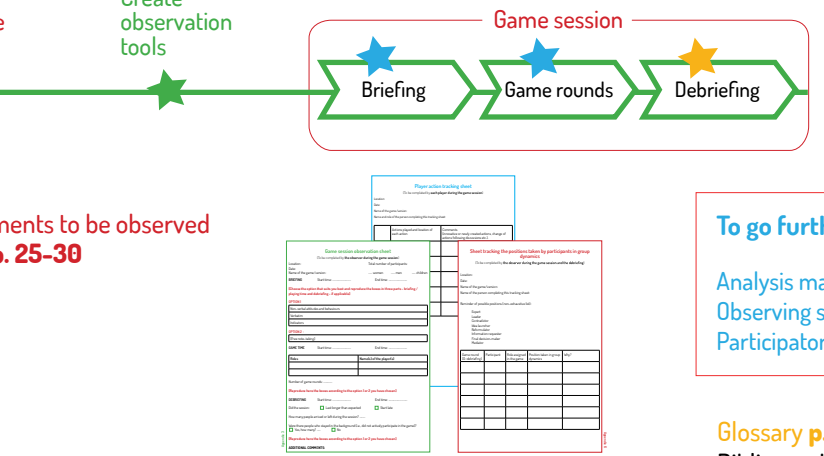

Attendance list Appendix 2

Game session observation sheet p. $\mathbf{3 4} \&$ Appendix 3

Player action tracking sheet p. $\mathbf{3 5} \&$ Appendix 4

Indicator tracking sheet $\mathbf{p .} \mathbf{3 6} \&$ Appendix 5

Sheet tracking the positions taken by participants in group

dynamics p. $\mathbf{3 7}$ \& Appendix 6

Ex-ante/ex-post questionnaire Appendix 7
To go further p. 49-51

Analysis made later on

Observing several game sessions

Participatory workshop [no game]

Glossary p. 52-53

Bibliography p. 54-55 







\section{What serious games are we talking about?}

Debates about the definition of a 'serious game' have stimulated the scientific community for almost 50 years (see Crookall, 2010). In this manual, we have chosen this simple definition:

An application (computerised or not) that combines serious aspects (teaching, learning, communication, marketing and information) and playful aspects (Alvarez, 2007; Schmoll, 2011).

The ComMod group mainly uses serious games in the form of computer simulations and/or role-playing games. These games represent and simulate socioecosystem dynamics (e.g. forest, watershed, livestock, fisheries etc.). By being around a game board or in front of a computer, several players interact and seek a solution to system problems (e.g. deforestation, poverty, water quality etc.) by learning together, coordinating and making collective decisions.

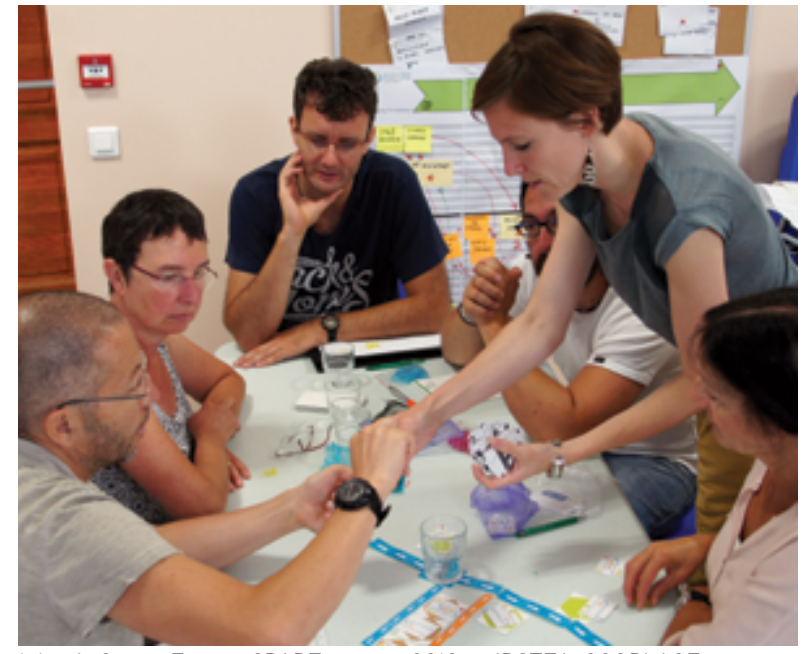

Wat-A-Game, France, SPARE project, 2016. @ IRSTEA-COOPLAGE

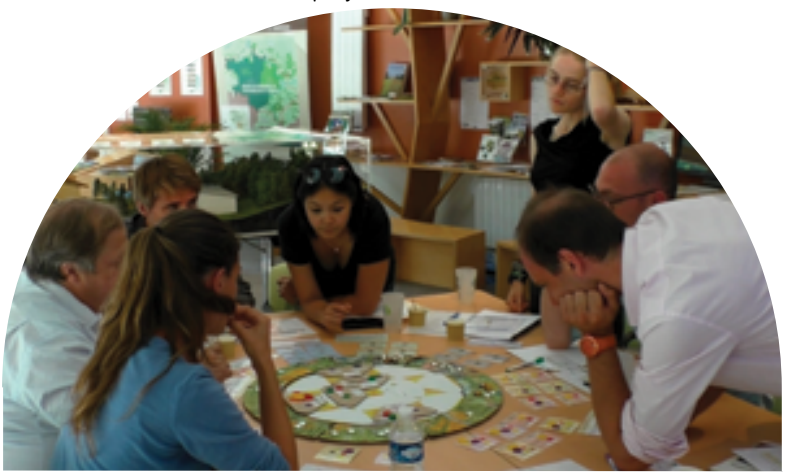

CapBiomass game, Fontainebleau Biosphere Reserve, CapBioTer project, 2018. Photo William's Daré 


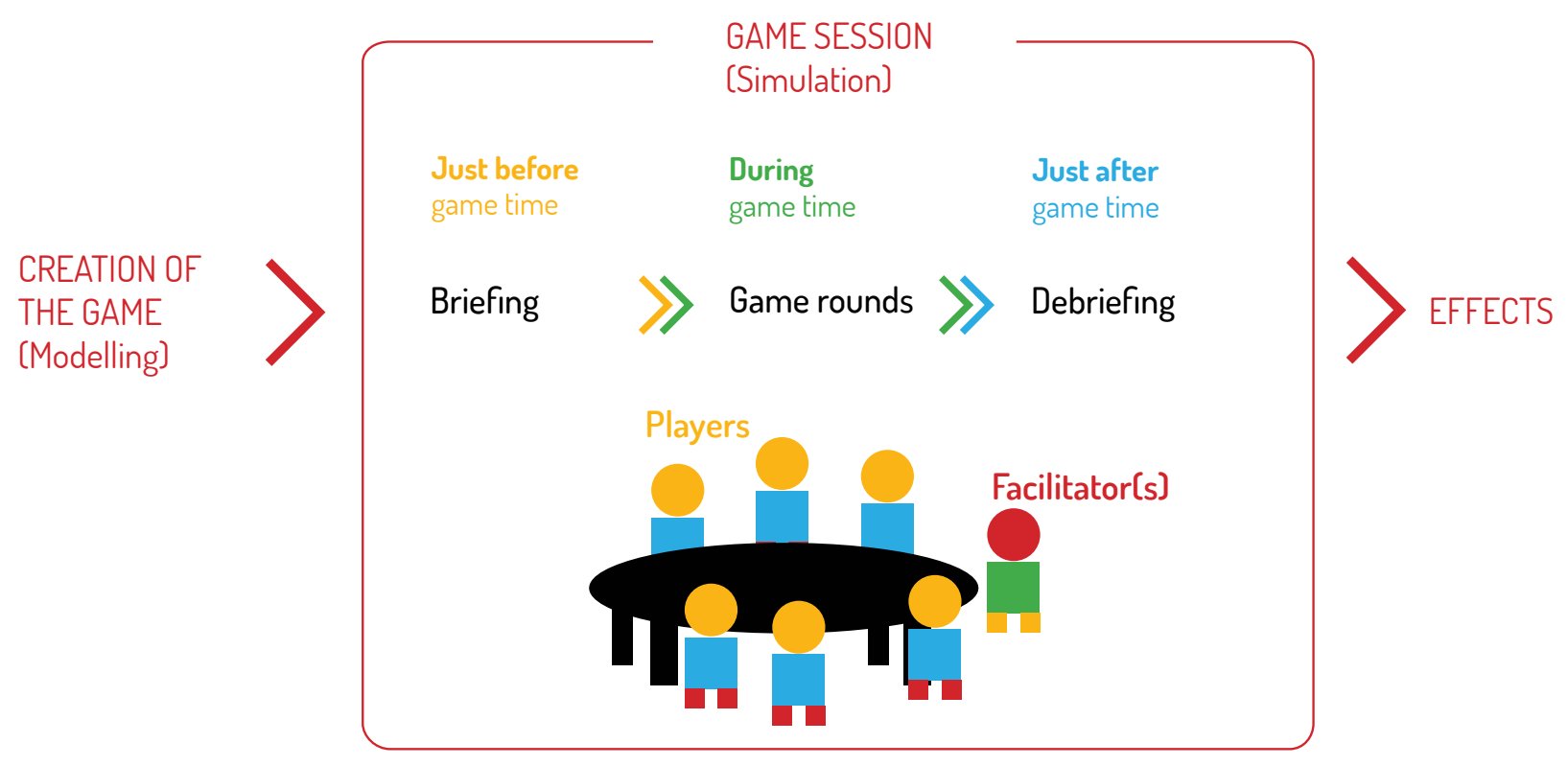




\section{In what context should this manual be used?}

This manual is about observing players and their interactions during a serious game session that meets the following five conditions:

1. The session focuses on the use of a game that has already been created that refers more or less abstractly to a given system. We deem a system to be a real situation that involves interactions between humans and nature (pastoralism in mountain areas, management of agricultural land, management of urban gardens, multi-use management of water etc.).

2. The number of players in the classroom is more than one.

3. At least one facilitator is present to facilitate interactions between players.

4. The course of the session follows the structure: Briefing - Game Turns - Debriefing.

5. In addition to the playful aspect, the objective of the session is to produce effects in relation to the system in question (new knowledge, conflict resolution, change of values, new practices, improvement of biodiversity etc.).

If this is not your case, this manual may not meet your expectations.

The figure opposite illustrates part of a classical ComMod process: from the conceptualisation of a model to the analysis of effects. In a complete ComMod process, there is the entire upstream part of the collective definition of the question through to the downstream evaluation of the effects, a stage which can lead to the emergence of a new problem leading to a new loop (cf. Methodological Guide, Daré et al. 2009). 


\section{Preliminary note on the ethical and legal aspects of data collection}

To the extent that the serious games mentioned in this manual involve the participation of physical persons, and that you are going to collect data on these persons, check which legislation and data protection rules your work, the participants and yourself are subject.

This is especially important with regard to:

- The image rights of the participants (if you are filming or photographing)

- The collection and use of personal or socio-demographic data collected

Be clear and transparent with the participants of the gaming session and other people involved.

In particular, be sure to specify the objectives of the observation and analysis of the data collected, the legal basis used, the persons responsible for the analysis, the persons who may have access to the data, the nature of the data collected and their degree of sensitivity, the length of time the data will be kept and the data security measures put in place. 


\section{Why observe a serious game session?}

The serious games we are talking about here are intended to produce effects on the participants in the session: for example, changes in practice or the acquisition of new knowledge. It is therefore necessary to be able to account for these effects through an observation protocol.

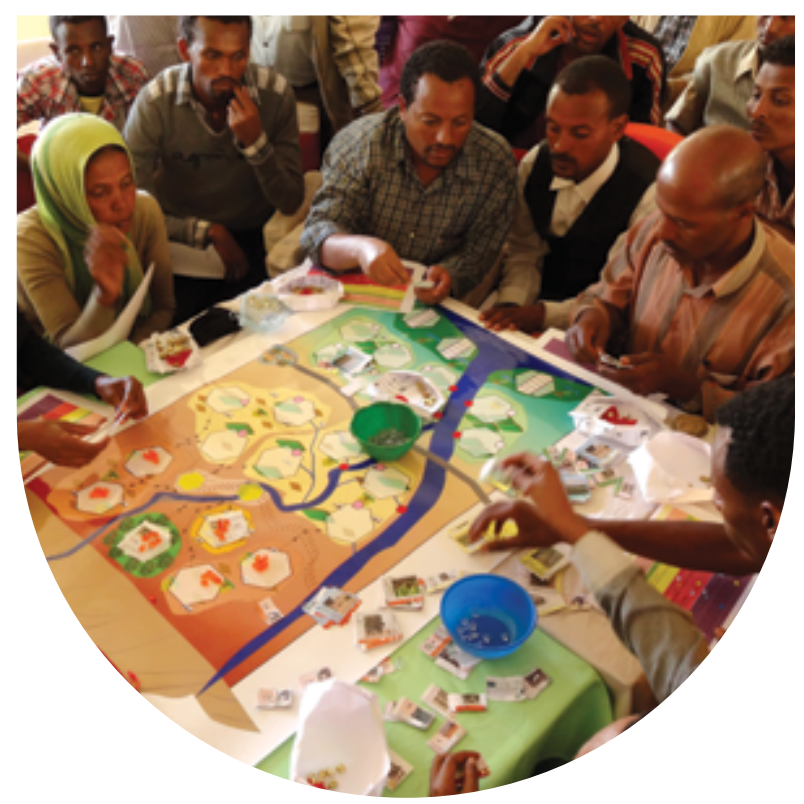

Game on grazing and erosion in Fogera, Ethiopia, AfroMaison project, 2013. (c) IRSTEA-COOPLAGE
Observation allows for the collection of data that will be used to:

- Characterise the game session (date, place, number of participants etc.)

- Report on the facilitator's influence on the game and players

- Understand the evolution of players' behaviours and relationships between them

- Enrich and contextualise the debriefing

- Evaluate the effects of participating in the game session (learning, practice, relationships etc.)

- Perform statistical analysis and/or session comparison 


\section{What data are we talking about?}

These data are of different kinds: qualitative (verbal and non-verbal) and quantitative (indicators).

Verbal data are of two kinds:

Verbatim data is a complete reproduction of what the players say.

Example: "You can't do this!"

Synthetic notes are summaries of observed elements.

Example: "The players decide to create a committee." The entire discussion leading up to the decision to form a committee is not recorded.

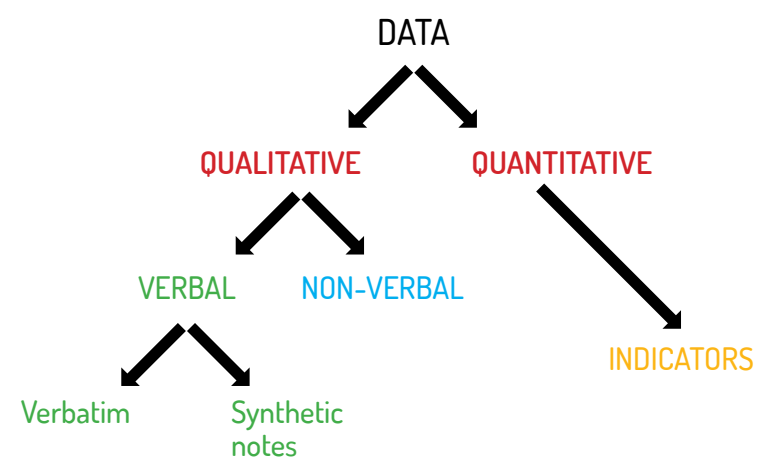

Non-verbal data correspond to body language, i.e. physical attitudes, facial expression, gestures, clothing, variation in tone of voice etc.

Examples: looking, smiling, yawning, nodding, leaving the room, standing in the centre of the group, pointing, whispering, shouting etc.

Indicators are numbers used to provide information about the value of a quantity. Examples: state of a resource, money obtained by the various players, number of times a word or gesture is used etc.

Later, we will discuss 'data collection' which to us is all of the observer's activities in recording the various elements above. 


\section{What equipment should I use to observe?}

Different materials can be used to collect data, continuously (video, audio) or discreetly (photos, notes, computer input).

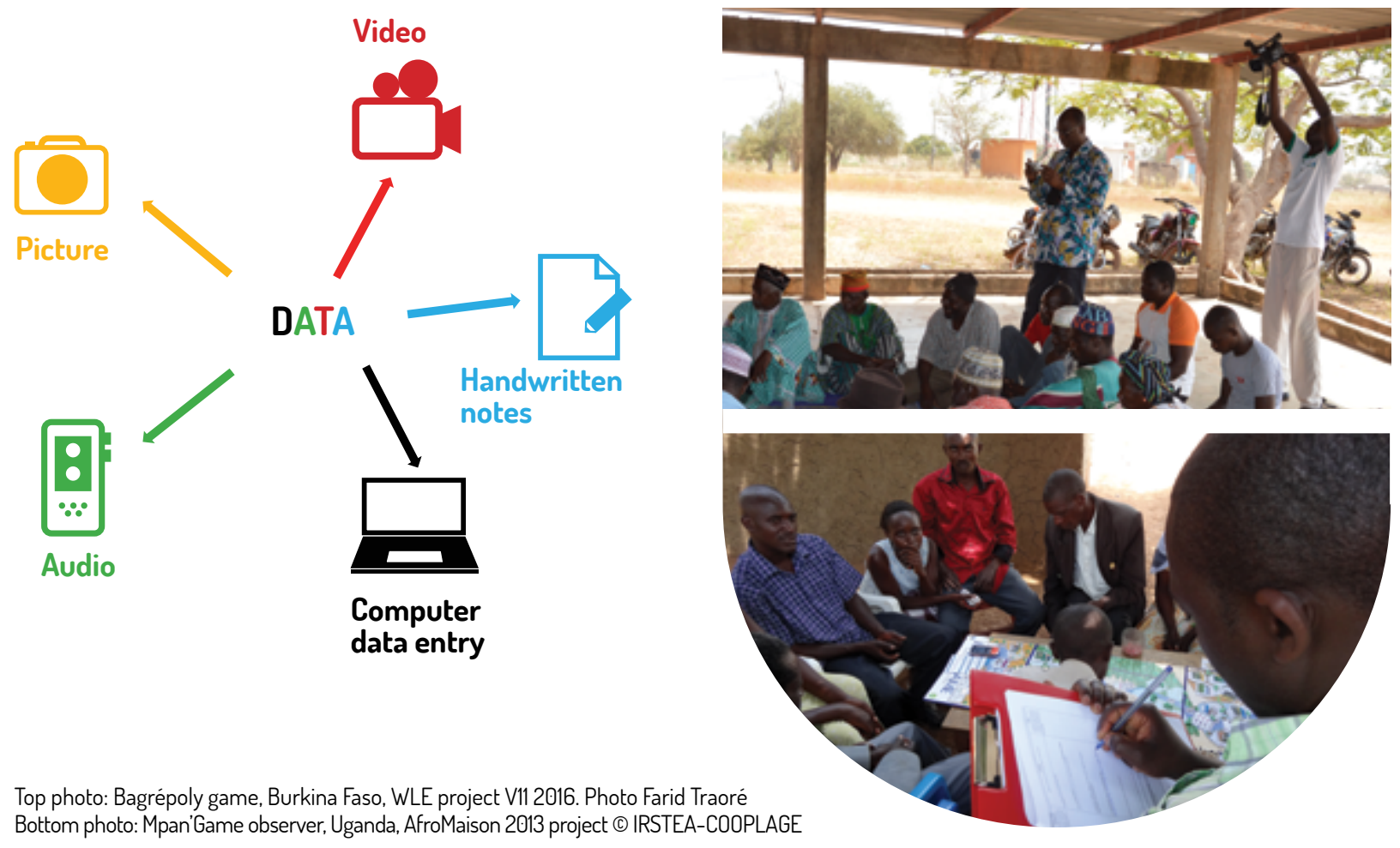


The game session involves different people in different roles:

- The facilitator is the one who hosts the game session, presents the rules of the game, organises the session, encourages dialogue and maintains the playful atmosphere. He/ she is the 'master of the game'.

- The players are the participants in the session who are active during the game rounds.

- The observer is in charge of collecting data on the game session and provides the elements that are observed to enrich the debriefing. Depending on the situation, the observer may be asked to facilitate the debriefing.

- The technician is in charge of the technical recording of the players' actions (for example, he or she will be the one who manipulates the computer software if necessary and the one who takes photos or videos).
Observation is mainly carried out by the observer. However, the players, facilitator(s) and technician(s) may also participate in data collection (see figure on page 32 ).

Depending on the objective of the session and the human resources available, the roles of facilitator, observer and technician may be carried by the same person.

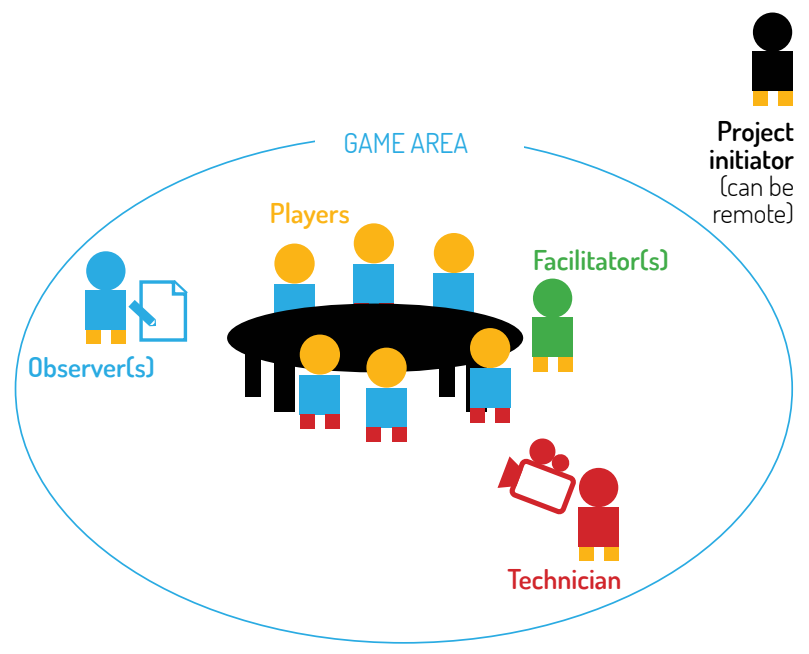




\section{What posture is appropriate for the observer?}

In this manual, we consider that a suitable posture is that of observer-as-participant or participant-asobserver:

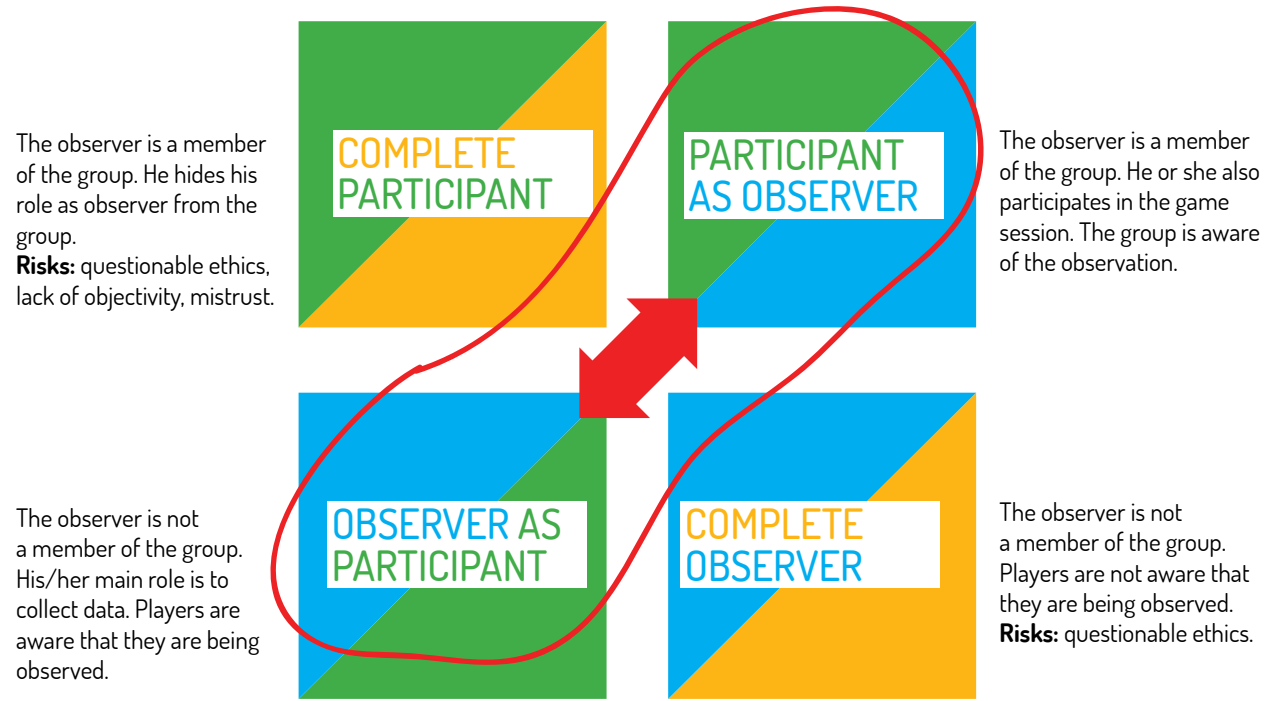

(Jankowski, 2012 based on Gold, 1958 and Adler \& Adler, 1987)

In both cases, observers make their role of observation transparent to the other players. They participate in the game session either by playing (participant-as-observer) or by mobilising the data collected during the debriefing (observer-as-participant). We consider that this participation is necessary in order to understand the representations and behaviours of the players. 


\section{When to observe?}

The observation starts at the beginning of the session (briefing) and continues until the participants leave (end of debriefing). The nature of the data collected varies according to the time of the game session.






\section{How to build the observation protocol?}

We suggest a five-step observation protocol:

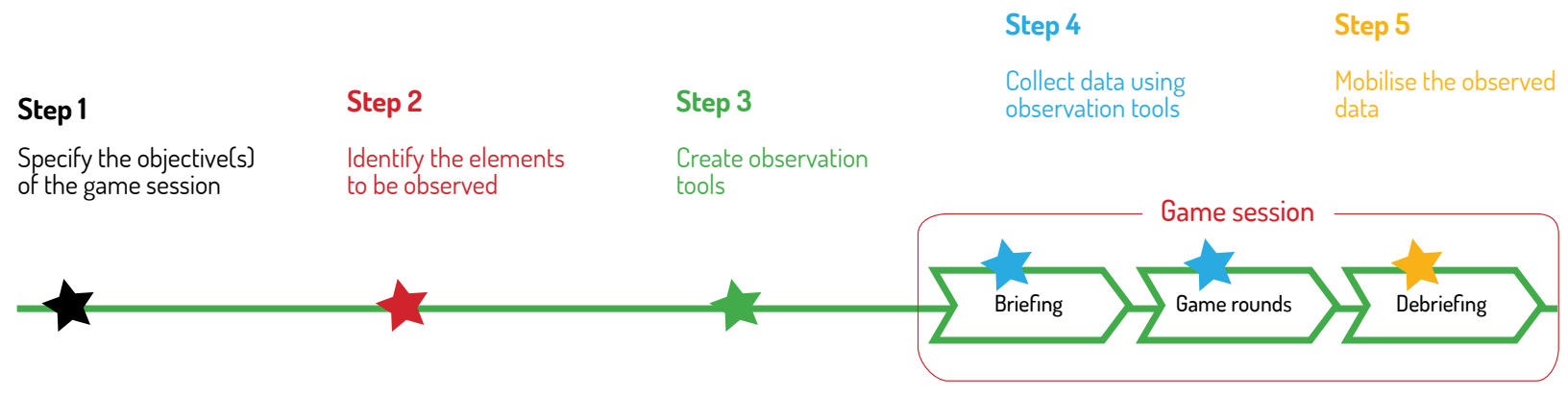

The more the observation protocol is thought about upstream, the greater the capacity to anticipate the observations to be carried out. However, we need to be careful to always remain open to surprises, i.e. unanticipated gaming effects or situations. 


\section{Step 1: Specify the objective(s) of the game session}

Here we are in a context where the game has already been built to meet a global objective.

Nevertheless, each game session may have its own objective. For example, the first session may aim to test or improve the game and the following sessions may aim to encourage players' learning.

Here we have identified six distinct session objectives that are neither exhaustive nor exclusive. These objectives have been identified on the basis of the experiences and work of researchers using roleplaying games (Etienne, 2011; Ferrand \& Daniell, 2006; COOPLAGE 2020).

\section{Enhancing knowledge transfer and learning}

The objective of the session is for players to acquire new notions, knowledge or concepts. This objective includes educational gaming sessions. It also includes learning about the method itself (i.e. role-playing).

\section{Getting values expressed}

The objective of the session is for players to express their preferences, what they consider right or wrong, just or unjust, sacred, fair etc.

3. Exploring new individual strategies (stimulating the emergence of new practices) The objective of the session is for the players to imagine new ways to implement their activities and/or propose new actions. 
4. Stimulating the emergence of new organisations and institutions

The objective of the session is for players to discuss new rules (management, resource allocation, sanctions, incentives, laws etc.) and/or propose new collective organisations (e.g. committee, association, network, cooperative, industry etc.)

\section{Promoting relationships between players}

The goal of the session is for each player to understand the point of view and the constraints of the other players, to create new interpersonal relationships and to reduce tensions.

6. Testing, validating or improving a game

The objective of the session is to see if the game is representative of the real dynamics, if the allocation of resources is balanced, if it is fun and interesting for all players etc.

Objectives of the game session

1 Enhancing knowledge transfer and learning

2 Getting values expressed

3 Exploring new individual strategies (stimulating the emergence of new practices]

(4) Stimulating the emergence of new organisations and institutions

5 Promoting relationships between players

6 Testing, validating or improving a game 


\section{Step 2: Identify the elements to be observed according to the session's objectives}

The following tables guide the observer on what to observe according to the session's objective(s) specified above (see p. 22-23). The elements to be observed are classified into three categories: nonverbal, verbal and indicators.

For each element to be observed, the observation tool that can be used is specified via the following four icons (see also p. 31):

- Game session observation sheet;

- Player action tracking sheet;

- Indicator tracking sheet;

- Follow-up sheet of positions taken by participants in the group dynamics.

When several tools are listed for the same item, the observer can choose the tool that seems most suitable. 


\section{Objective 1. Promote knowledge transfer and learning}

\section{NON-VERBAL elements to be} observed

OBS Use of artifacts (board, pawn, board, graphs) by some players.

E.g. player drawing a graph to follow the evolution of a resource on his sheet.



\section{Change of actions} ACT performed by players. E.g. As a result of information given by the facilitator or a discussion with another player, a player changes the action that he or she originally planned to do.

\section{VERBAL elements to be observed}

Sentences expressing:

OBS Knowledge, perceptions (and their evolution).

E.g. I didn't think it was that difficult to...; I didn't know that my action had such a consequence; I didn't know that the environment reacted in such a way; I understood...

OBS

The comprehension questions asked by the players about the dynamics of the system and the explanations provided to answer them.

E.g. In which direction does the water flow?

\section{INDICATORS to be observed}

IND Status of one or more resources.

IND Financial status of players.

OBS Number of questions asked about the operation of the system.

OBS Counting of the qualitative elements expressed in the two previous columns.

E.g. Number of times the verb 'to know' or 'to understand' is expressed.
Step 
Objective 2. Getting values expressed (equity, justice, sacred, preferences, good/bad)

\section{NON-VERBAL elements to be observed}

OBS Physical signs of approval or disapproval.

E.g. nodding, smiling, grouping of like-minded players, patting on the back etc.

Gestures of reference to the sacred.

E.g. signs of prayer before an action in the game, blowing on the dice before throwing them etc.

OBS

Illegal, forbidden, beyond the rules of the game, actions of mutual aid, compassion, set up in the collective interest, or simply surprising from the observer's point of view
VERBAL elements to be observed

OBS Sentences expressing value judgments.

E.g. I didn't think I was able to cheat, it's fair/unfair, you have no right to do that, I chose from..., it's not respectful of the elders, it's not fair, you're selfish!

INDICATORS to be observed

IND OBS

Number of offending players

IND Financial status of players who show an interest in accumulating

IND OBS

Count of the qualitative elements expressed in the next column, e.g. number of times the word 'just' or 'equity' is expressed, religious references expressed etc. 
NON-VERBAL elements to be observed

OBS ACT New actions or performed by players.

E.g. when a player who had planned to play an action to maximise his income opts instead for a pro-environmental action following information given by the facilitator.
VERBAL elements to be observed

OBS Sentences expressing changes in practices or proposals for new practices.

E.g. maybe I could do..., I'm going

to test..., I've never done this before...
INDICATORS to be observed

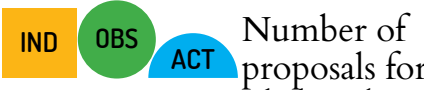

new practices made by players. 


\section{Objective 4. Stimulating the emergence of new organisations and} institutions

\section{NON-VERBAL \\ elements to be observed}

OBS Lawful or unlawful arrangements reflected in the actions, attitudes, groupings or evictions of players.

E.g. corruption via bribes, signing of an agreement etc.

\section{VERBAL elements to be observed}

Sentences expressing:

OBS The spontaneous creation of a collective (group, committee, associations, network, cooperatives etc.)

E.g. if we shared this, if we got together etc.

OBS

Decisions on management rules or ACT resource allocation, management plans, sanctions, incentives, new laws.

OBS The relationships between individuals and the collective to which they belong in the game.

E.g. players from one village interacting with another village in conflict because of their neighbourhood on the perimeter.

OBS POS Identification of a representative to attend a meeting.

OBS

IND The exchange of resources among players. E.g. loan of money, seeds etc.

\section{INDICATORS to be} observed

IND Count of the qualitative

elements expressed in neighbouring column. E.g. number of times a player is proposed as a representative, number of resources exchanged (money etc.), number of sanctions or rewards. 


\section{NON-VERBAL elements to be}

\section{observed}

OBS Appearance of signs of empathy. difficulty E.g. lending money to a player in

OBS POS Appearance of new roles in the game or new positions in the group. E.g. a leader who decides, a player who gets up to speak in front of the group, a player who moves to get closer to another one, a player who moves backwards in a corner, who gets excluded by the group etc.

OBS Formation of groups facilitating exchanges between some (possibly at the expense of other players).

E.g. size, collective, isolation of certain players, formation of a network (distributed, centred on certain players) etc.

OBS Positions of individuals in the gaming space reflecting privileged exchanges between some players, and the exclusion of others.

OBS Use of artifacts (board, pawn, board, graphs) by some players to explain something to other players.

\section{VERBAL elements to be}

observed

Sentences expressing group dynamics, power games:

OBS Conflicts, tensions, collaboration, cooperation, solidarity, trust.

OBS

Use of collective pronouns (we, you, they) to express the formation of groups or subgroups and the expression of a sense of belonging or exclusion.

E.g. here's what we can do etc.

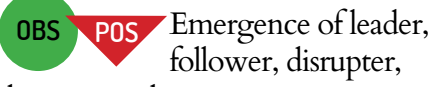

decision-maker, etc.

E.g. you don't let me etc.

OBS Understanding, interest in others. E.g. I understand better why $X$ is reacting this way; why are you saying this?

OBS Opinion on others. E.g. I didn't think he was like that, I don't agree with, you wouldn't want to, can someone etc.
INDICATORS to be observed

OBS IND Monitoring of the sharing of speeches (balanced or not): number of speeches and length of time each person speaks. players in difficulty (who could not perform the desired actions).

OBS IND Counting of the qualitative

elements expressed in neighbouring column. E.g. interactions between such and such a player, number of times the pronoun 'we' is used, number of players who have lent money to another player etc. 


\section{Objective 6. Testing, validating or improving a game}

NON-VERBAL

elements to be observed

OBS Signs of interest or disinterest.

E.g. Yawning, signs of trouble; players moving in or out of the playing area; players standing/ sitting; active/passive players; smiles/laughs.

\section{VERBAL elements to be observed}

OBS Sentences expressing:

- Interest/disinterest

E.g. I had a good time, I find it interesting, it's the first time I'm taking part in this kind of workshop.

- Suggestions for improvement

- Blockages or incomprehension

- Membership/rejection

E.g. it's great what we just did, I never thought of exchanging with such and such a person, it sucks, I didn't learn anything, that's why etc.

- The link with reality (market dynamics, calibration of resource dynamics).

E.g. Your price evolution is not realistic! It represents our daily life so well!

- A request to continue the experiment or on the contrary to stop it.

- A request to invite others.

\section{INDICATORS to be}

observed

IND Unexpected imbalance of indicators.

E.g. distribution of resources.

OBS Count of the qualitative

elements expressed in neighbouring column (smiles, sentences etc.).

OBS

IND Number of players in

difficulty (who were unable to perform the desired actions). 


\section{Step 3 : Create the observation tools}

The items to be observed in the tables on pages 25-30 can be recorded using various tools.

\begin{tabular}{|c|c|c|c|}
\hline Phase & Observation tool & Appendix & Icon \\
\hline \multirow[t]{3}{*}{ BRIEFING } & Game session description sheet & Appendix 1 & \\
\hline & Attendance list & Appendix 2 & \\
\hline & Ex-ante questionnaire & Appendix 7 & \\
\hline \multirow[t]{3}{*}{ GAME ROUNDS } & Indicator tracking sheet & Appendix 5 & IND \\
\hline & $\begin{array}{l}\text { Sheet tracking the positions taken by participants in the group } \\
\text { dynamics }\end{array}$ & Appendix 6 & \\
\hline & Player action tracking sheet & Appendix 4 & \\
\hline \multirow[t]{2}{*}{ DEBRIEFING } & $\begin{array}{l}\text { Sheet tracking the positions taken by participants in the group } \\
\text { dynamics }\end{array}$ & Appendix 6 & \\
\hline & Ex-post questionnaire & Appendix 7 & \\
\hline \multirow{2}{*}{$\begin{array}{l}\text { ENTIRE GAME } \\
\text { SESSION }\end{array}$} & Game session observation sheet & Appendix 3 & \\
\hline & Video, audio, photos, computer recording & & \\
\hline
\end{tabular}


Collective serious games observation manual

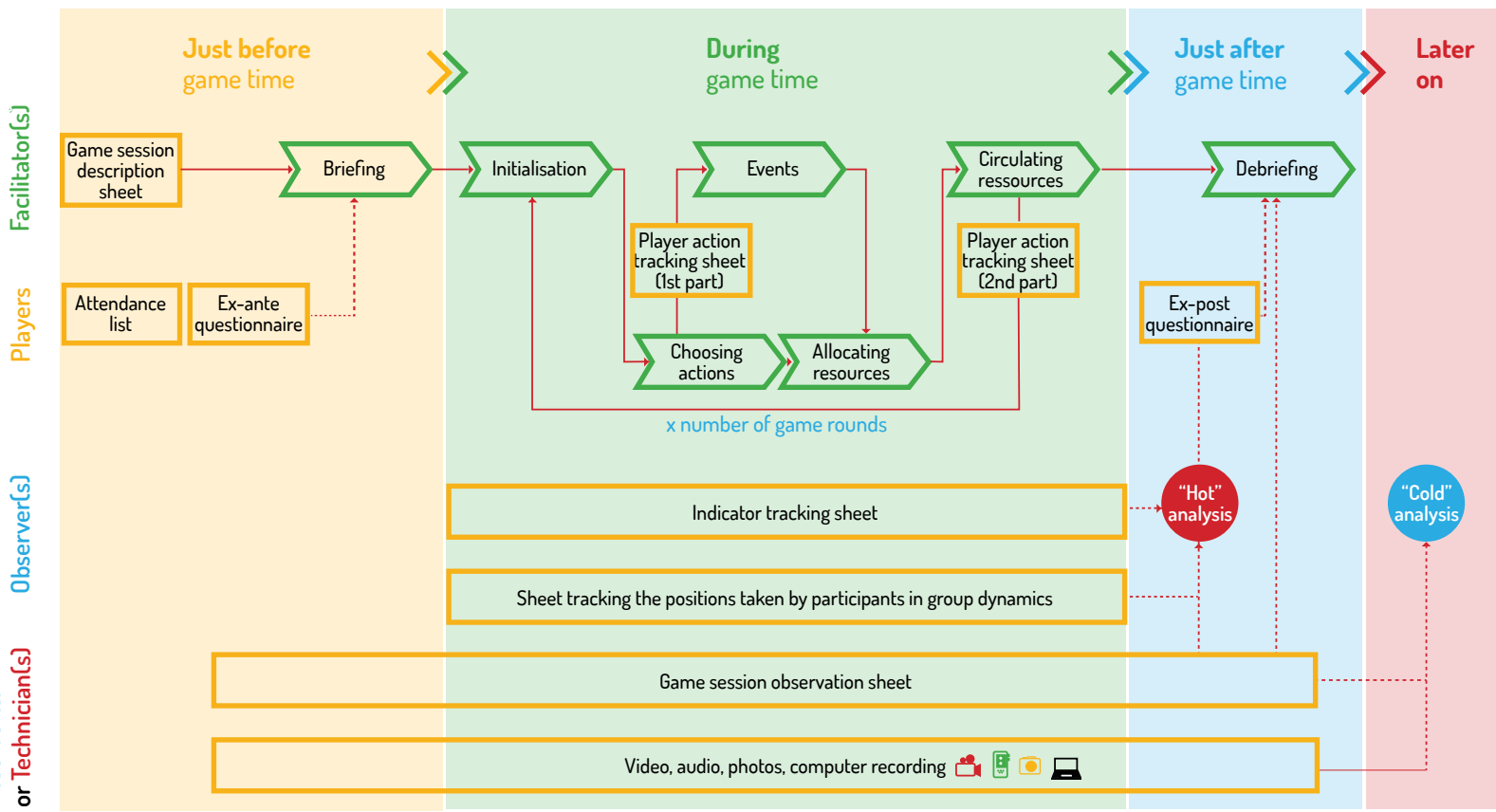

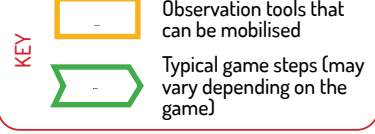


Whatever the objective(s) of your game session, we advise that you systematically complete the following forms:

- Game session description sheet

- Attendance list

For the other tools (sheets or questionnaires), their use and content depend on your objectives.

All of these tools are suggested as indications to help you bear in mind the elements which need to be observed and eventually ensuring that your observation skills become automatic. As your experience grows, you will find that you have more freedom to produce your own tools.

For each of the session's objectives, see the corresponding tables on pages 25-30. In these tables you will find the elements that should be observed. For each item, we have indicated with an icon the tool you can use to observe it. For example, if you see the ACT icon in front of 'Player action changes', this means that the 'Player action tracking sheet' tool is used to observe these changes. Video, audio and photo recordings allow you to observe all the non-verbal elements. 


\section{Game session observation sheet (from briefing to debriefing)}

This is the central tool for observing game session. Depending on your observation experience, there are several options for completing the session observation sheet:

Option 1: Make a box corresponding to each of your objectives and write down the elements to be observed in each box as a reminder (see OBS icon in the tables on pages 25-30)

- Advantage: The information will then be much easier to mobilise for hot debriefing, especially when there is little time between the gaming session and the debriefing.

- Disadvantage: This can be difficult to do during the session, as it involves having to keep in mind the elements corresponding to each objective.

Option 2: Make three separate boxes for non-verbal, verbal and indicators (if relevant).

- $\quad$ Same advantages and disadvantages as Option 1.

Option 3: Take notes 'on the fly' on blank paper.

- Advantage: Easier to do for the observer during the session.

- Disadvantage: Requires time between the game session and debriefing to reorganise the notes taken.

These three options require the observer to have in mind the different elements to be observed. If your team comprises several observers, you can divide up the elements to be observed (for example, verbal for observer 1 and non-verbal for observer 2). 


\section{Player action tracking sheet}

This consists of reporting on the actions carried out by each player in each round of the game. If you want players to comment on their choice of actions, remember to create a comment space for this (see Appendix 4).

You can also ask them for other information if necessary (e.g. "Why did you change your actions between the two rounds?") but be careful not to include too much information, as this work is tedious for the players and can take them out of the fun atmosphere.

Think beforehand about the most appropriate time to fill in the monitoring sheet. This can be done:

- At the beginning of the round, just after each player has chosen the actions he or she wants to perform.

- At the same time the observer fills out the indicator tracking sheet.

- At another time to be defined. 


\section{Indicator tracking sheet}

Begin by identifying the indicators to be observed (see tables on pages 25-30).

Be careful not to identify too many indicators, as monitoring them may be difficult.

Consider whether or not you want to computerise the indicator tracking sheet. Be careful though, just because the sheet is computerised does not mean that you need to put in more indicators! Keep an eye on the time required for data entry, even if it is easier to produce summary graphs.

Specify who should fill in this sheet: facilitator (especially if there is a computer assistant), observer or player. Make sure that the indicator tracking sheet is clear and simple enough to be filled in easily.

Think ahead about the most appropriate time to fill in the indicator tracking sheet. Usually this is done at the end of a round, but for some games and some indicators it may be done:

- During a round: for example, for a game on a watershed, to distinguish between the upstream/middle and downstream balance; or if the round is divided into seasons or time periods.

- After several rounds: for example, for indicators related to reforestation that will only be relevant and visible after longer periods of time; or following particular events (weather, consultation meetings between players etc.) 


\section{Follow-up sheet of the positions taken by participants in group} dynamics

Regardless of their assigned role in the game, some participants are likely to take on particular positions within the group at some point, and/or change positions during the session.

These positions include, but are not limited to, the following:

- Expert

- Leader

- Opponent

- Idea initiator

- Reformulator

- Information seeker

- Final decision-maker

- Mediator

The tracking sheet allows you to record which participant is in which position at which time. 


\section{Step 4 : Collect data using observation tools}

Now that the observation tools have been built, it is time to use them to collect the data you need for your work. Divide the tasks and tools among the different observers and facilitators.

Before the game session begins, make sure that your observation protocol is adapted to the constraints of the workshop organisation:

\section{If you are a small number of observers relative to the number of} players

Delegate observation to the players as much as possible (e.g. by including more items to be observed in the player action tracking sheet). You can also focus your observations on certain categories of participants, certain roles or particular moments in the gaming session.

\section{If you have several sub-areas of the game, but a limited number of observers}

Choose a sub-space on which to focus your observation/writing. However, if equipment is available, you may film, photograph and/or record the other sub-spaces in order to use this data for cold analysis. 


\section{If you use the recording technique (audio, camera, video)}

Make sure that you have properly charged your equipment and that you have enough recording space on your device.

\section{If you are not sure how to take notes (observation sheet)}

You have two options

- Verbatim: Take notes of all players' words

- Synthetic note-taking: note down an event, a fact, without necessarily trying to transcribe literally the player's words (e.g. spontaneous creation of a cooperative: what interests us is that this cooperative was created in the game, not necessarily all of the discussion and verbatim records that led to its creation).

The observer alternates between these two types of note taking according to the objectives of the observation. For example, if the objective is to observe the emergence of new organisations, the observer takes a verbatim note as soon as the players discuss the creation of a collective (group, cooperative, internal organisation etc.). The rest of the discussions are taken in the form of synthetic notes.

Wadiga game, France, ComMod Research School, 2015. Photo William's Daré






\section{Step 5 : Mobilise the observed data}

\section{In the course of the game}

Indicators can be mobilised during game rounds to make players react to a given situation.

Remember to facilitate the production of synthetic graphs if necessary and to make the results visible to the players concerned.

The observer can use his or her observations to alert the facilitator to certain behaviours in the game (cheating, corruption, self-help etc.) or topics discussed in small groups so that the facilitator can discuss them if he or she wishes.

The observer can also alert the facilitator if he or she notices that players are bored, that some are being left out, or there are other imbalances in the evolution of the game..

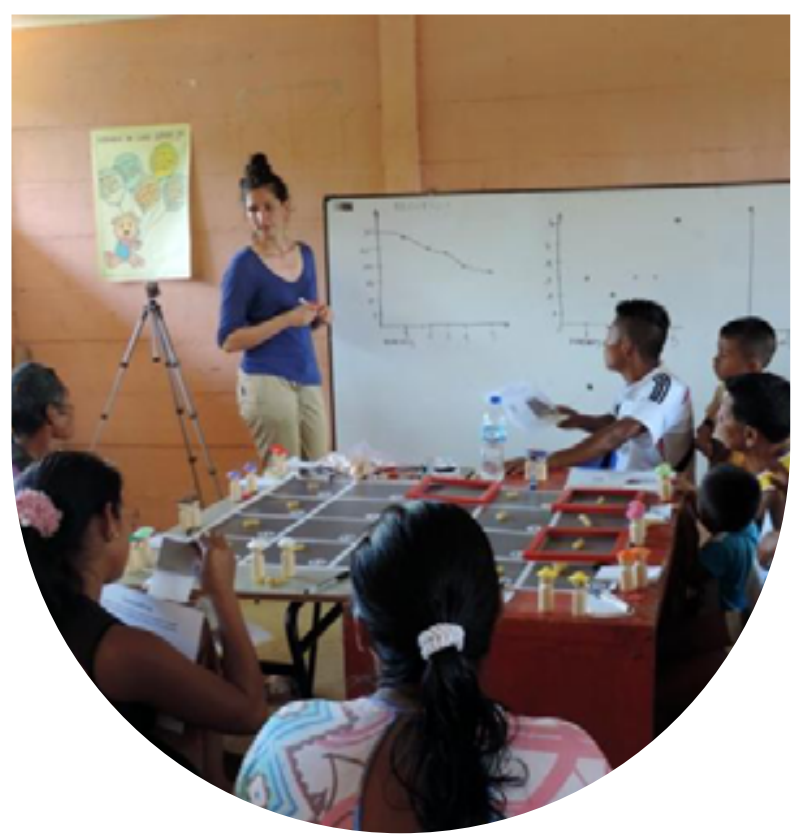

Use of synthetic graphs during a session of the Ticoya game in Colombia, 2017. Photo Maria Paola Quiceno Mesa 


\section{During the debriefing}

\section{What is debriefing?}

Debriefing is based on the idea that all group dynamics take place in an alternation between phases in which emotions (positive or negative) dominate and phases in which interactions focus on the accomplishment of the group's tasks.

However, these different phases and their sequence are difficult for group members to perceive. People outside the group of players (such as the facilitator, observer etc.) therefore help the group to better understand why and how this or that result was achieved.

The debriefing is when:

1. The players share their experience of the game session, sometimes lived individually or in sub-groups, with the rest of the participants.

2. The facilitator guides the return to the real situation, encourages the participants to look for new avenues for reflection or solutions.

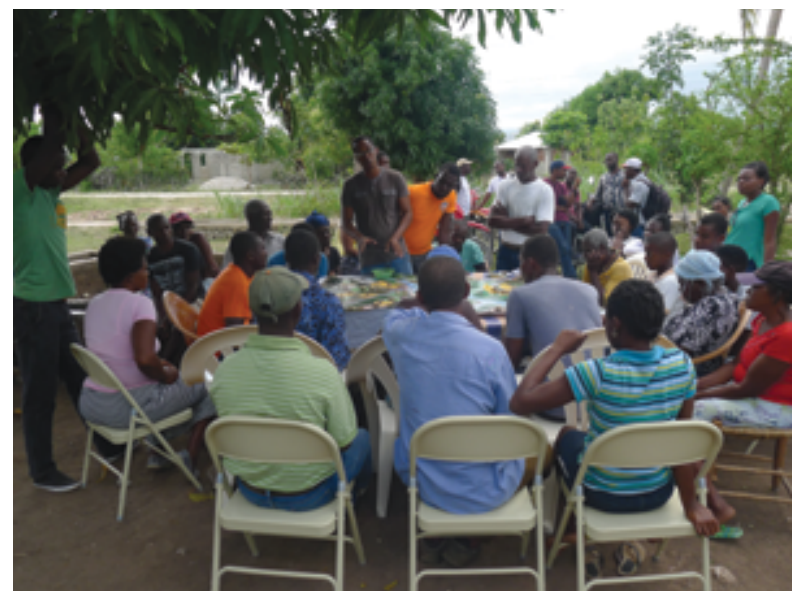

Debriefing of a Zacavé game session, Haiti, GSE-Avezac project, 2018. Photo Ola Dolinska 


\section{How is the debriefing organised?}

Debriefing is generally organised in five phases:

Emotions: Sharing the emotions felt by each participant. This first phase is essential to allow players to eventually get rid of negative feelings (resentment, anger, misunderstanding, failure etc.) that could limit their learning and their ability to reflect on the experience.

Events: Sharing of the progress of the entire session among all the players: tasks and actions carried out by players, game routines, highlights (curve/indicator monitoring), changes in pace due to events that occurred in the game etc.

? Explanations: The same fact can have several explanations. Here, we need to put them into perspective through the players' behaviours and the individual and collective strategies that led to these results.

$\rightarrow \cdot \leftarrow$ Link to reality: This involves going back over the links between the game and the real situation at the origin of the organisation of the session (convergences, divergences).

T Projection into the future: How the results of the game session will be used in the future. 
Each of these phases is conducted by the facilitator, who solicits feedback from the observer(s) to inform the players' opinions. From experience, the events, explanations and link to reality phases are often intrinsically linked in explaining an action situation that has arisen in the course of the game. For example, a player will mention: "I chose to plant wheat on my plot [event] because I didn't have enough money to plant sunflowers [explanation] and it's true that in reality that's what happened to me several years in a row [link to reality]."

The facilitator may choose to organise the debriefing by addressing several of these situations one after the other. The observer will then provide the observed elements specific to each situation.

Top photo: Preparation for the debriefing between observer and facilitator. Kabamukuni game, Botswana, Prosuli project, 2019. Photo Etienne Delay.

Bottom photo: Player summarising his strategy with other participants. Dai Prek game, Cambodia, ANR Doubt project, 2019. Photo Caroline Coulon.
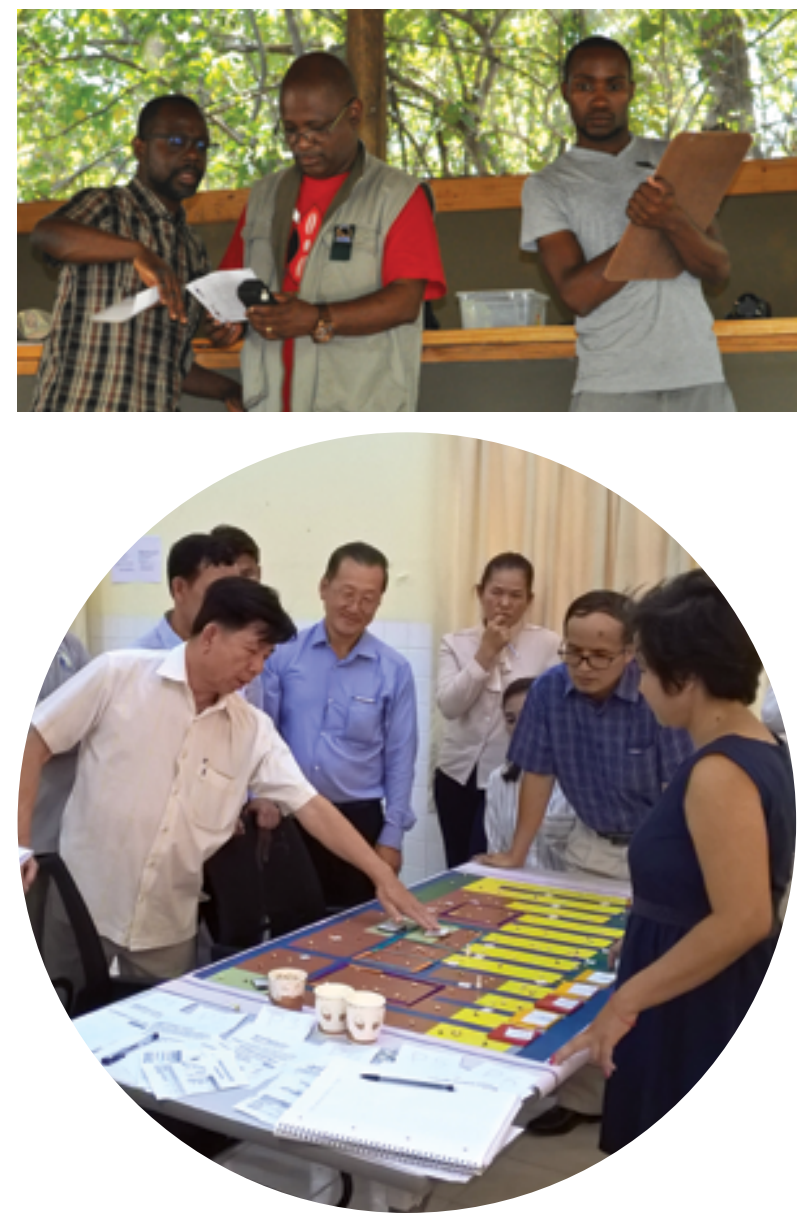


\section{How to mobilise the data observed during debriefing?}

This delicate phase requires organisation.

- Just before the debriefing, the observer and facilitator should take some time to organise the conduct of the debriefing.

- The observer should share with the facilitator the most relevant noted elements that he/she has identified either for each of the five phases (e.g. by highlighting them with different colours on the observation sheet, or by putting a letter indicative of the corresponding step next to them), or according to themes (or action situations) that the facilitator wishes to address (by listing them clearly).



- The observer helps the facilitator to conduct the debriefing by providing additional elements to go beyond the simple description of the progress made by players during the session.

- In principle, players are given the floor first. The observer then intervenes to enrich the discussion based on his or her observations. 


\section{Emotions}

Your observations make it possible to:

- Report on the atmosphere of the game session. Using your observation sheet, you can share the words that reflect the different emotional phases the group went through during the different rounds of the game.

- Put into perspective the individual emotions expressed by each person. This will show, whatever these emotions are, if they were collectively overcome and allowed the group to progress towards solving the tasks.

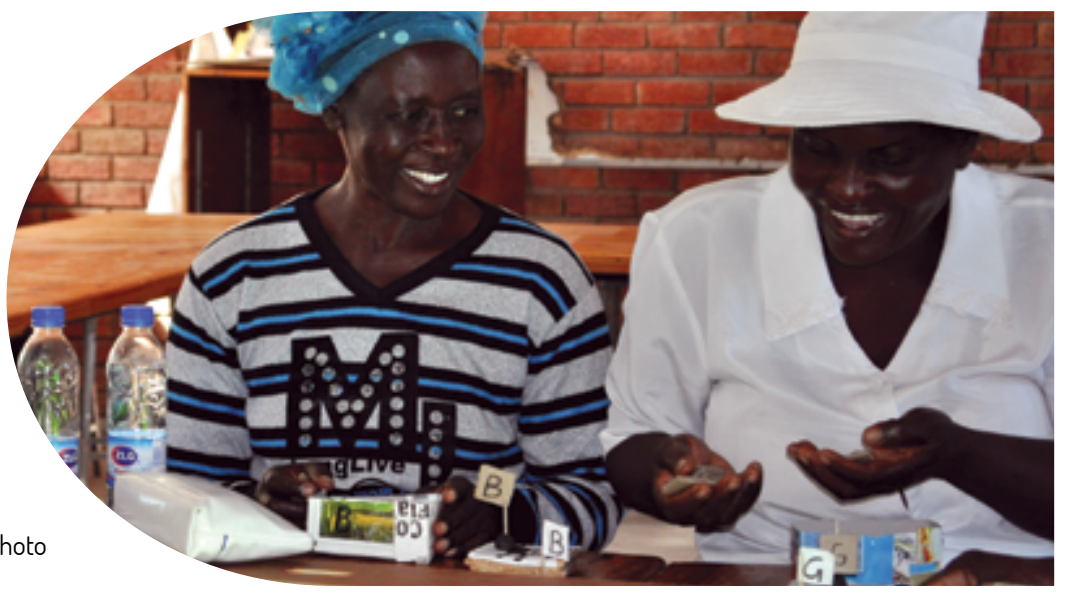




\section{Events Explanations?}

When the facilitator introduces the general dynamics of the game session, players react to their own actions in the game (based on their action tracking sheet, if necessary), reflecting their understanding of the mechanisms and thereby justifying their choices.

The observer intervenes, based on his or her observation sheet, to:

- Illustrate what the players say.

- Question the knowledge expressed and the different justifications given by the players during the game to explain their choice to others.

- Report on non-verbal interactions between players: setting up sub-groups, at what time, at whose initiative, to do what etc.

- Clarify the redirections made by the group: for example, by explaining how certain tensions observed between individuals or sub-groups or the introduction of events may have led the group dynamics (new decision, new grouping, etc.) in a particular direction.

You should pay particular attention to highlighting the changes you have perceived in terms of value, preference and justification. Your observations should be linked (as much as possible) to the dynamics of the game (by identifying the turn in the game and the possible link to the evolution of indicators).

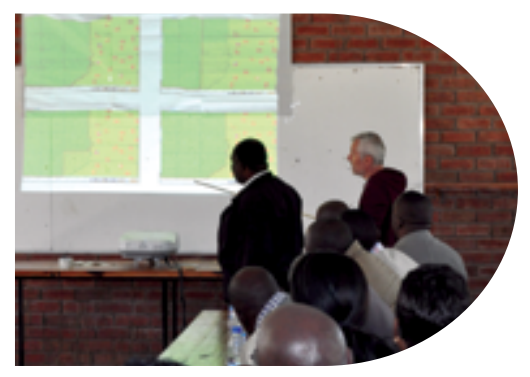

Graphic explanation of game phases Kulayinjana game, Zimbabwe, Dream project, 2017. Photo William's Daré 


\section{Link to reality}

Your role here is to report on all the references to reality made by the players during the game.

The observer intervenes, based on his or her observation sheet, to:

- Underline the convergences and divergences that players have highlighted between the game and reality. To do this, you should rely mainly on the players' verbatim statements ("Normally it doesn't happen like this!"), in particular by sharing the moments when the terms real, reality, realism, real life etc. were used. You can also go back over the surprises and unexpected events that appeared during the game session because they often reflect elements of reality that were not considered in the design of the game.

- Mention when players justified their actions in the game by a link to reality: "I did this because in real life...”.

- Highlight any link to reality made unconsciously or not expressed by the players: e.g. if a player turns to a neighbour in real life to obtain a loan when the rules of the game led him or her to turn instead to the player playing the role of banker.

\section{Projection into the future $\nabla^{\circ}$}

This phase is generally not based on observational data since it is a projection into the future. However, if players mentioned during the gaming session elements related to such a projection (e.g. "Next time I make a decision in my job, I will do it differently"), it is relevant to mention them. 


\section{Overview of the steps to follow}

\begin{tabular}{|c|c|c|c|c|}
\hline & & & $\begin{array}{l}\text { Step } 4 \\
\text { p. } 38-39\end{array}$ & $\begin{array}{l}\text { Step } 5 \\
\text { p. } 40-47\end{array}$ \\
\hline $\begin{array}{l}\text { Step 1 } \\
\text { p. 22-23 }\end{array}$ & $\begin{array}{l}\text { Step } 2 \\
\text { p. 24-30 }\end{array}$ & $\begin{array}{l}\text { Step } 3 \\
\text { p. 31-37 }\end{array}$ & $\begin{array}{l}\text { Collect data using } \\
\text { observation tools }\end{array}$ & $\begin{array}{l}\text { Mobilise the } \\
\text { observed data }\end{array}$ \\
\hline
\end{tabular}

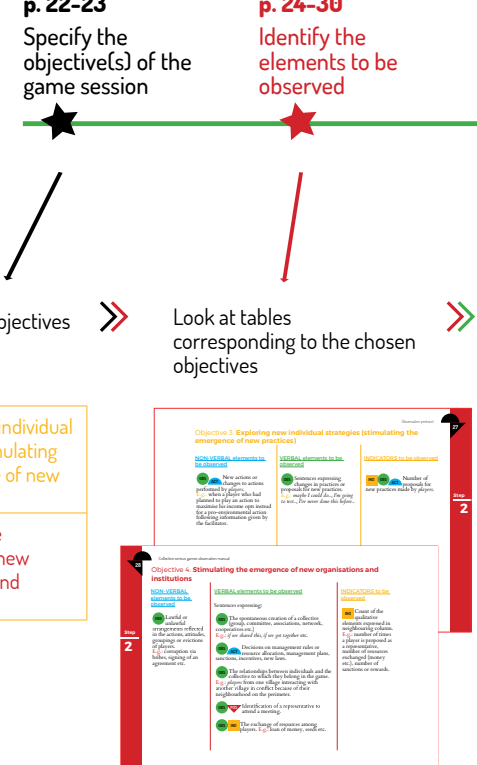

Create

observation

tools
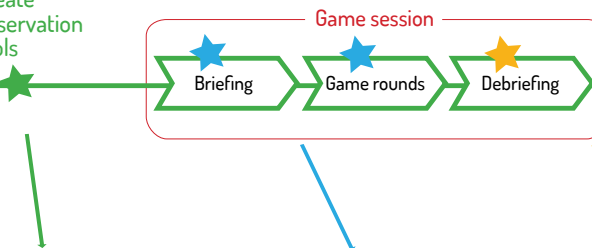

Chose your objectives \》

Look at tables

corresponding to the chosen

E.g. For the preparation of the $\gg$

game session observation

sheet, use the tables in step 2

to list all the elements to be

observed that you consider

relevant and that are

associated with the icon $0 \mathrm{BS}$
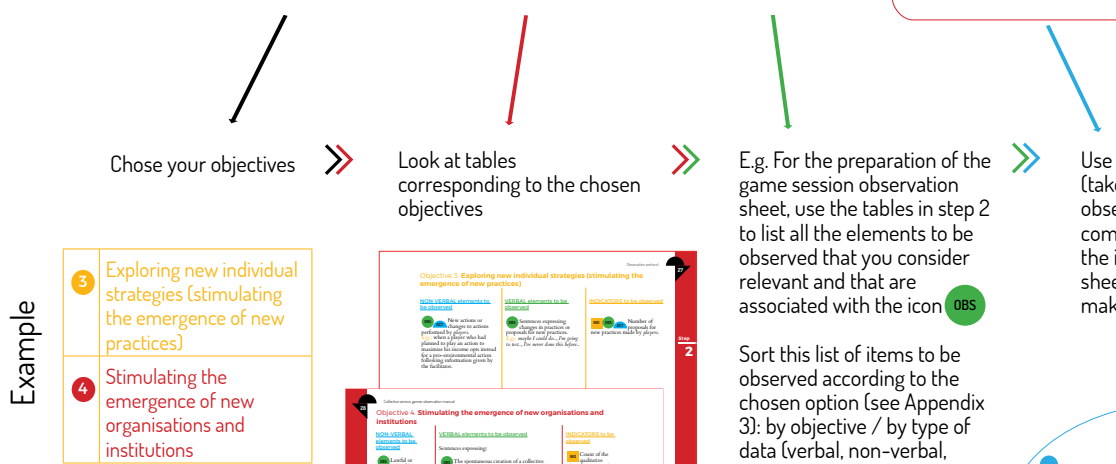

Sort this list of items to be

observed according to the

chosen option (see Appendix

3): by objective / by type of

data (verbal, non-verbal,

indicators] / free note-taking]

and then transfer this list to

the corresponding boxes. This

will serve as a memo when

taking notes.

Use observation tools

take notes on the

observation sheet,

complete indicators on

the indicator tracking

sheet, take photos,

make videos etc.J.
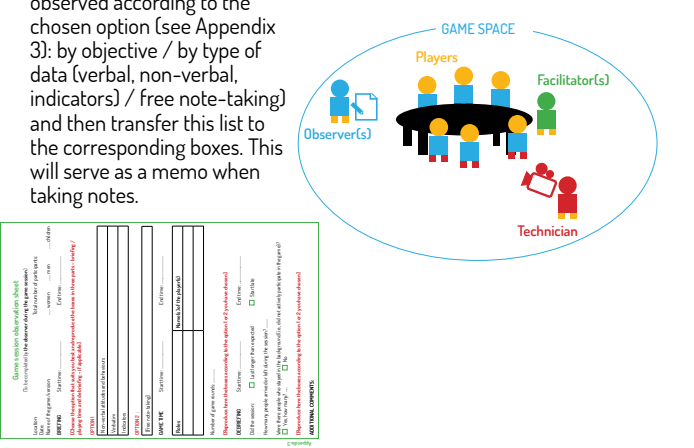

Look at and summarise

your notes:

During the game to

make players react to

their situation in the

game or alert the

acilitator.

\section{During the debriefing} to supplement players' comments and enrich

the discussion with

your observations. 


\section{For further developments}

\section{If you wish to mobilise your observations for a cold/a posteriori analysis}

All the data collected through the different tools can be analysed in more detail, for example for research purposes or to report on the effects of your gaming session on your system. This is called cold analysis and can be of a qualitative, quantitative or mixed nature.

Video, audio and photo recordings and ex-ante/ex-post questionnaires are useful for cold analysis. Their time-consuming analysis is difficult to perform between game rounds and debriefing.

-

- 1 Verbatim transcription of video or audio recordings can be used for speech analysis.

- The analysis of players action follow-up sheets can be used to establish a grid of the different strategies of the players via statistical processing.

- $\square$ The analysis of ex-post/ex-ante questionnaires (see Appendix 7) can, for example, provide additional information on players' knowledge and values (for example, by asking them to express their preferences directly in addition to observing how these are expressed in the game through their discourse and behaviour). They can also be used to analyse changes. 
The definition of an analysis framework, the use of transcription software and manual transcription/ translation can be considered for this. You can consult online courses on textual analysis to help you process the information collected to answer your questions (e.g. OpenClassrooms, CNAM, CIRAD CIHEAM etc.).

\section{Be careful not to drown yourself in recordings!}

$\square$ Remember to define beforehand how you intend to use recorded data. Think well in advance about the complementarity between the media used: e.g. if you only make audio recordings of group discussions, you won't be able to easily find out who said what afterwards. However, you can do this in addition to video or observation. Finally, some players are uncomfortable with being filmed, so ask yourself in advance if you will actually reuse the video for your analysis.



Transcription and translation of the audio and video recordings of game sessions, India, Assam project, 2016. Photo Swen Bos 


\section{If you want to observe more than one game session}

In this manual we focus on one game session. Of course, if you want to observe a succession of game sessions as part of a longer process, simply repeat the protocol offered here. You can then aggregate the data, make comparisons between different groups, report on changes over time etc.

\section{If you want to observe a participatory workshop (without games)}

If you are observing interactions between participants that do not involve a game (e.g. a participatory planning workshop, a participatory model design workshop, a working meeting etc.), you can base your approach on this manual because a number of the elements to be observed (especially nonverbal ones) may be similar. Others, however, may not be relevant.

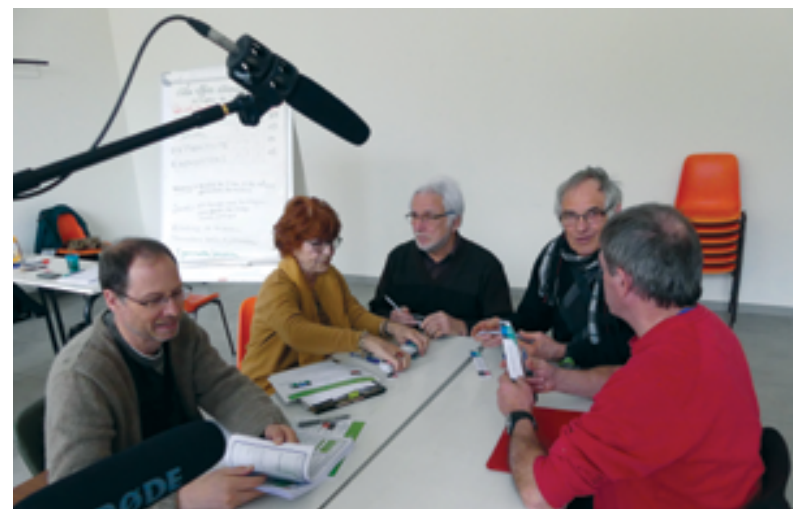

Observation of a participatory planning workshop on the River Drôme, France, SPARE project, 2016. @ IRSTEA-COOPLAGE-SMRD 
Briefing: The moment just before game time when the facilitator welcomes the participants, introduces the session's objective and organisation, and explains the elements of the game.

Cold analysis: Interpretation of data observed after the end of the game session for research purposes or to report on long-term effects.

ComMod: An association whose main goal is to design, analyse, develop and promote scientific research and its applications in the field of Companion Modelling, including its main methods and tools, namely role-playing, multiagent modelling, and social simulation (https:// www.commod.org). ComMod also refers to the Companion Modelling approach.

Debriefing: The moment just after game time when the facilitator speaks with players to get them to comment on the experience they have just lived. This is the moment when you come out of the play phase and return to the real situation that led to the serious game.
Facilitator: Person who leads the gaming session, presents the rules of the game, organises the session, encourages dialogue and maintains the playful atmosphere. He or she is the 'master of the game'.

Game time: The time during which participants discover the components of the game and interact with each other. Game time is usually composed of several rounds of play.

Indicators: Numbers used to provide information about the value of a quantity. E.g. state of a resource, money in the possession of various players etc.

Monitoring and evaluation: A systematic investigation of an object that can be carried out for a variety of purposes, by a variety of stakeholders and at various times (based on Chess, 2000).

Observation: The act of looking closely at phenomena, events and people in order to 
study them, understand them, record them, draw conclusions from them etc. (adapted from Dictionnaire Larousse)

Observation tool: Techniques or procedures used to obtain and collect data about the gaming session. E.g. observation sheets, questionnaires, indicator tracking sheets etc.

Observer: A person who is responsible for collecting data on the game session and who provides the observed items to enrich the debriefing. Depending on the situation, the observer may be asked to facilitate the debriefing.

Players: Session participants who are active during the game rounds.

Posture: The moral attitude of the observer as reflected in the emotional, social, philosophical and political position he or she takes in the game space and within the group of players. Their posture influences their way of observing (according to Daré et al., 2011).

Serious game: An application (computerised or not) that combines serious aspects (teaching, learning, communication, marketing, information) and playful aspects (Alvarez, 2007; Schmoll, 2011).

Synthetic notes: Summaries of observed elements. E.g. "Players decide to form a committee": the entire discussion leading up to the decision to form a committee is not recorded.

System: Real situation involving interactions between people and nature (pastoralism in mountain areas, management of agricultural land, management of urban gardens, multi-use water management etc.).

Technician: Person in charge of the technical recording of players' actions (e.g. he or she will be the one who manipulates the computer software if necessary, the one who takes photos or videos).

Verbatim: Complete reproduction of what the players say (the whole sentence is written without changing it). 


\section{Bibliography}

Adler, P.A., \& Adler, P. (1987). Membership roles in field research: Vol. 6. Qualitative research methods. Newbury Park, CA: Sage Publications.

Alvarez, J. (2007) PhD Thesis Du jeu vidéo au serious game: approches culturelle, pragmatique et formelle. Université de Toulouse 2. https://hal. archives-ouvertes.fr/tel-01240683

Bachelet, R. (2012) Course "Designing and administering a questionnaire ». Ecole Centrale de Lille. http://rb.ec-lille.fr/l/Analyse_de_donnees/ Methodologie_Conception_et_administration_ de_questionnaires.html

Chess, C. (2000). Evaluating Environmental Public Participation: Methodological Questions. Journal of Environmental Planning and Management, 43(6), 769-784.

COOPLAGE (2020) Coupling Open and Participatory Tools to Let Stakeholders Adapt for Water Management. http://cooplaage. watagame.info
Crookall, D. (2010) Serious Games, DeBriefing, and Simulation/Gaming as a Discipline. Simulation \& Gaming 41(6) pp. 898 -920 https://doi.org/10.1177/1046878110390784.

Daré, W., Barnaud, C., d'Aquino, P., Étienne, M., Fourage, C. \& Souchère, V. (2011) The commodian stance: interpersonal skills and expertise. In Etienne M., (2011)

Dufour, C. (2007) L'ABC de l'élaboration d'un questionnaire. Intervention au $34 \mathrm{e}$ Congrès annuel de l'ASTED 7-10 novembre 2007 http:// dufour.ebsi.umontreal.ca/asted2007_cdufour.pdf

Etienne, M. (2011). Companion modelling $A$ participatory approach to support sustainable development. Edited by M. Etienne. Versailles, France: Editions Quae.

Ferrand, N., \& Daniell, K. A. (2006). Comment évaluer la contribution de la modélisation participative au développement durable? In Séminaire DDT. Lille, France. 
Gold, R.L. (1958). Roles in sociological field observations. Social Forces, 36(3), pp. 217-223

Jankowski, F. (2012) Course " Participatory observation. From the anthropological field to interdisciplinary and participatory environmental research ». CEFE/Université de Montpellier.

Schmoll, P. (2011) Jeux sérieux : exploration d'un oxymore. Revue des Sciences sociales, Presses Universitaires de Strasbourg, 2011, Jeux et enjeux pp. 158-167. https://hal.archives-ouvertes.fr/hal01300938/

SUP Structure Universitaire de Pédagogie Université Toulouse III Paul Sabatier (2006) "Guide d'élaboration de questionnaires d'évaluation des connaissances " https://sup.ups-tlse.fr/ pedagogie/outils/qec.htm\#3 


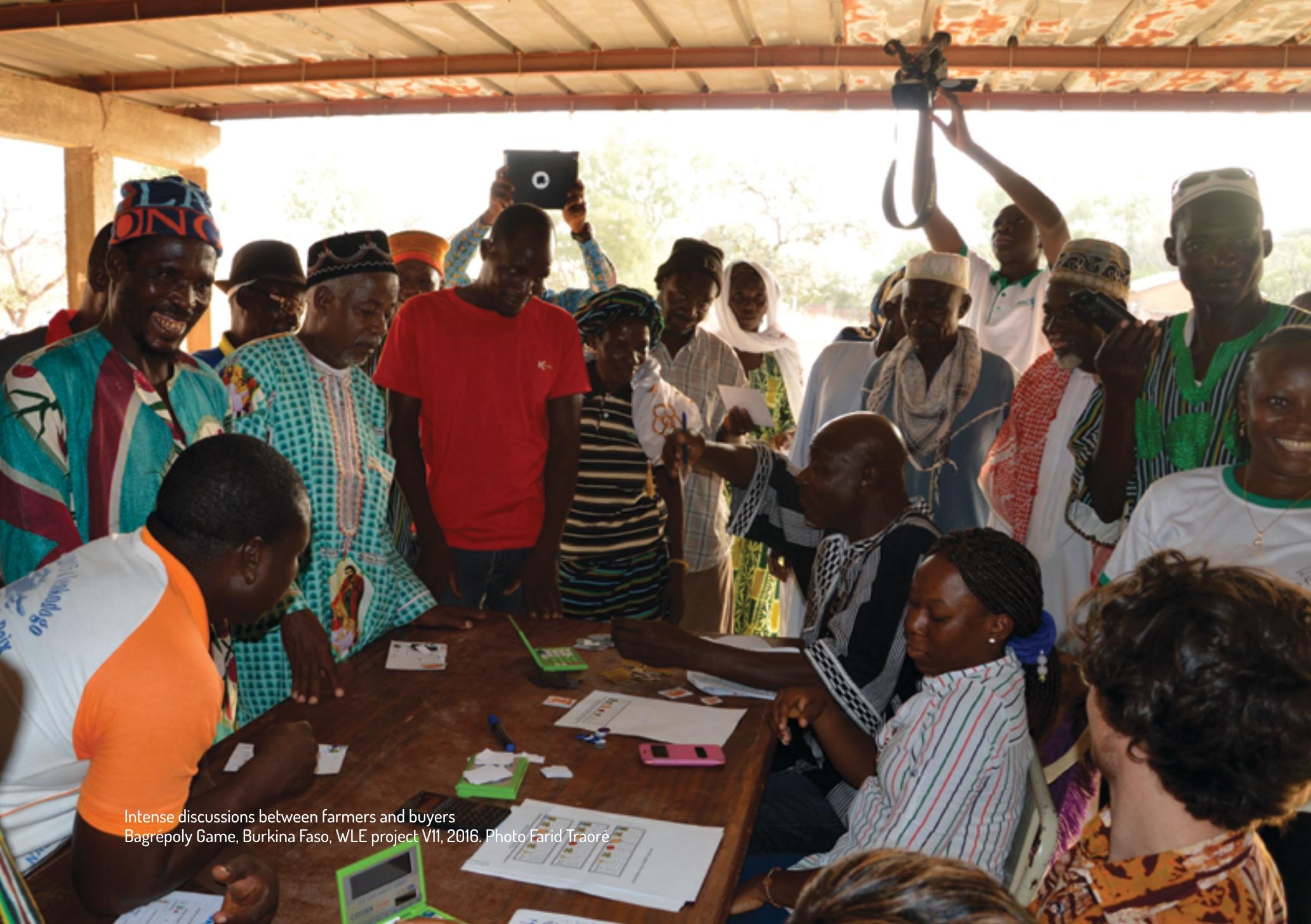




\section{Appendices}

Appendix 1 Game session description sheet

Appendix 2 Attendance list

Appendix 3 Game session observation sheet

Appendix 4 Player action tracking sheet

Appendix 5 Indicator tracking sheet

Appendix 6 Sheet tracking the positions taken by

participants in group dynamics

Appendix 7 Ex-antelex-post questionnaire 







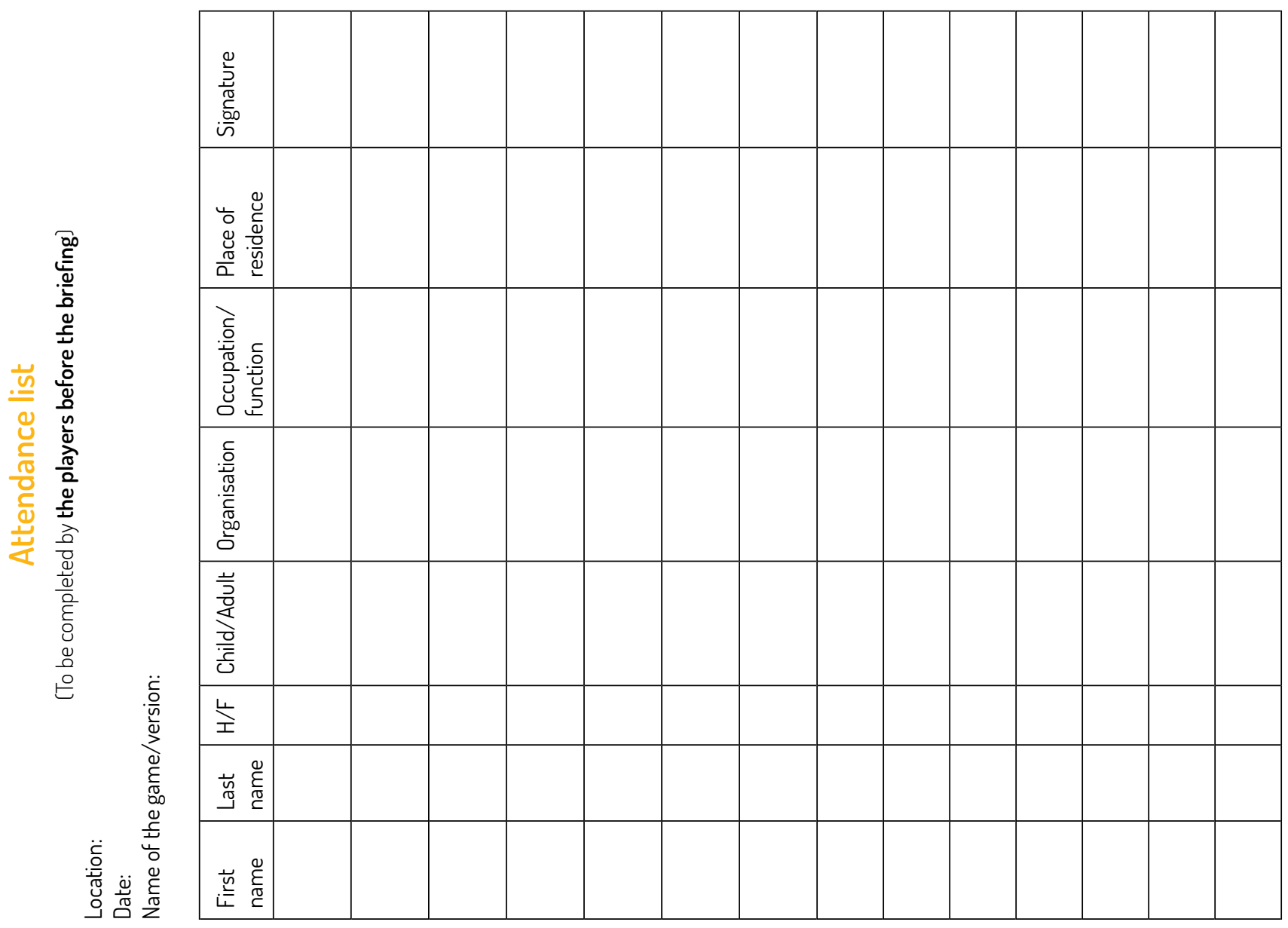




\section{Appendix 3}

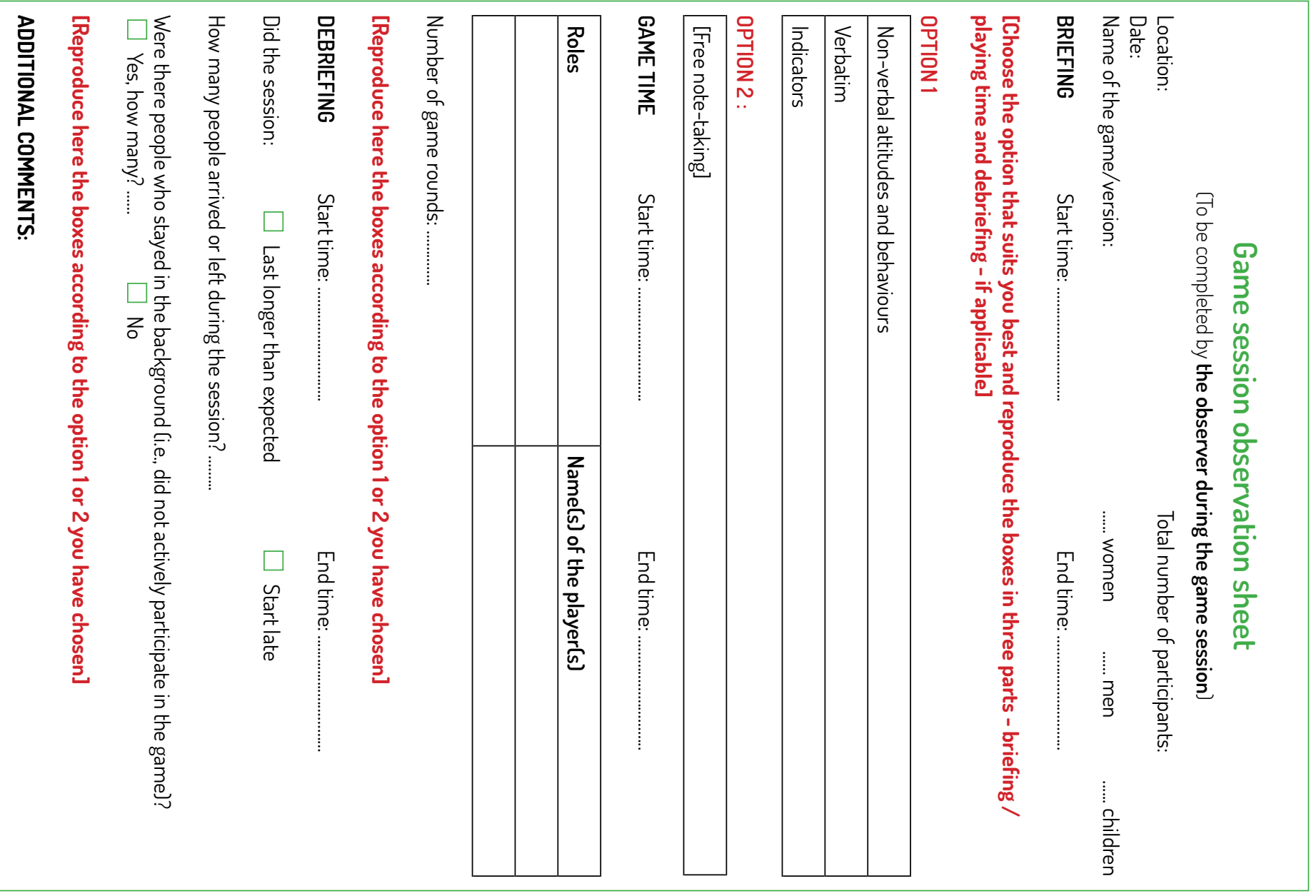




$$
\|_{n}
$$




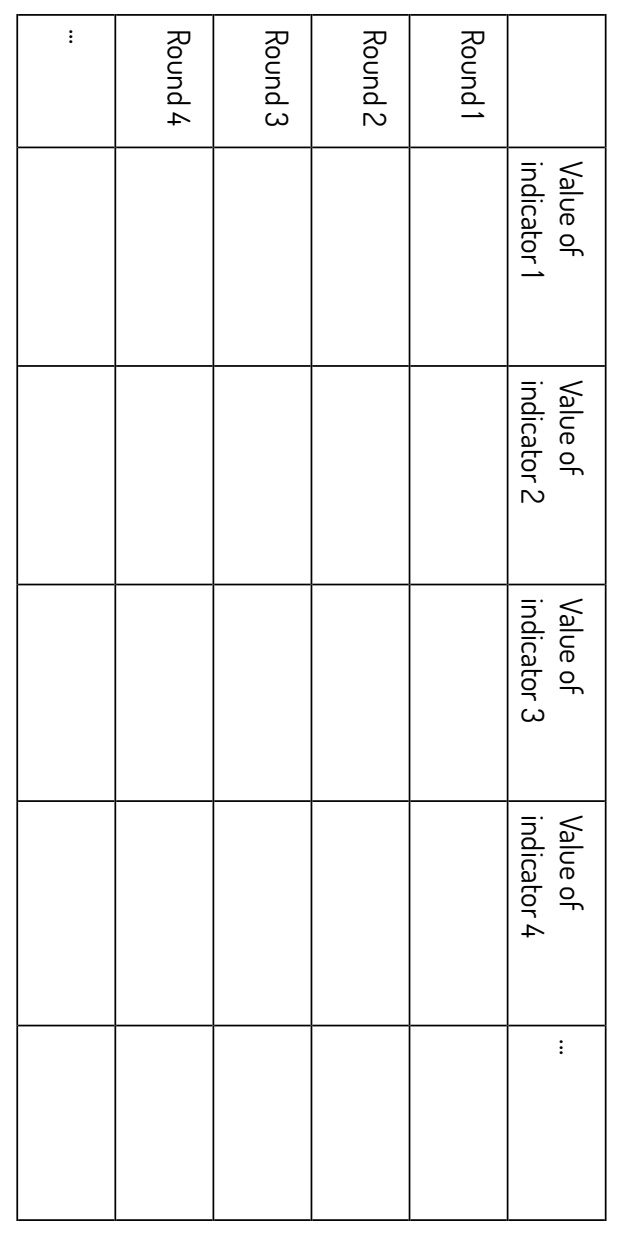

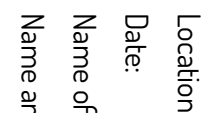

을 움

음

일

空

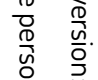

윽



궁
응
8

$\frac{3}{3}$

$\frac{\sqrt{8}}{\frac{8}{8}}$

察

움

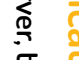

容

ฏ.

气.

욱 즉

으

궁

흔

克

ำ

돈.

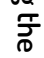

学

ఠ

芯. 

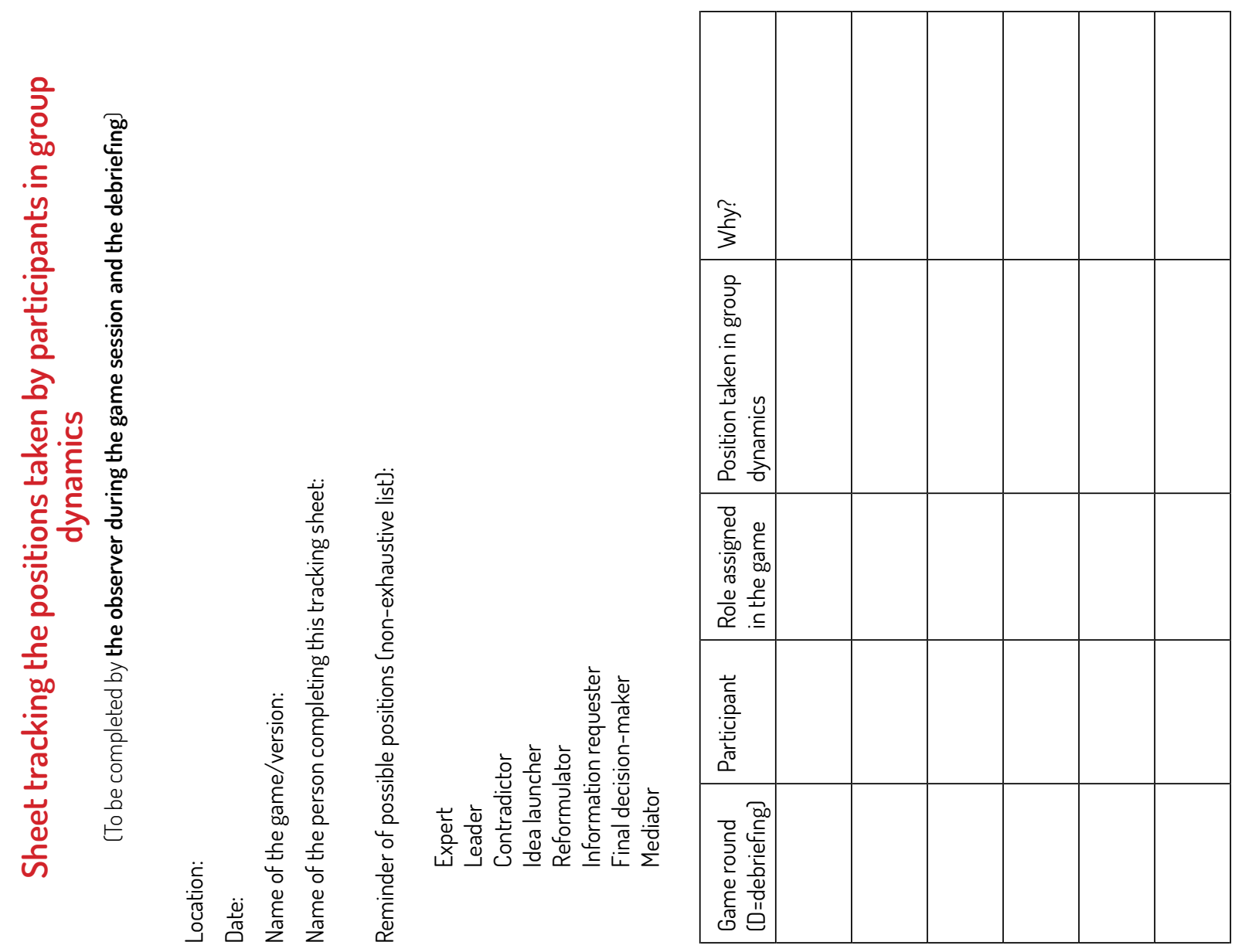


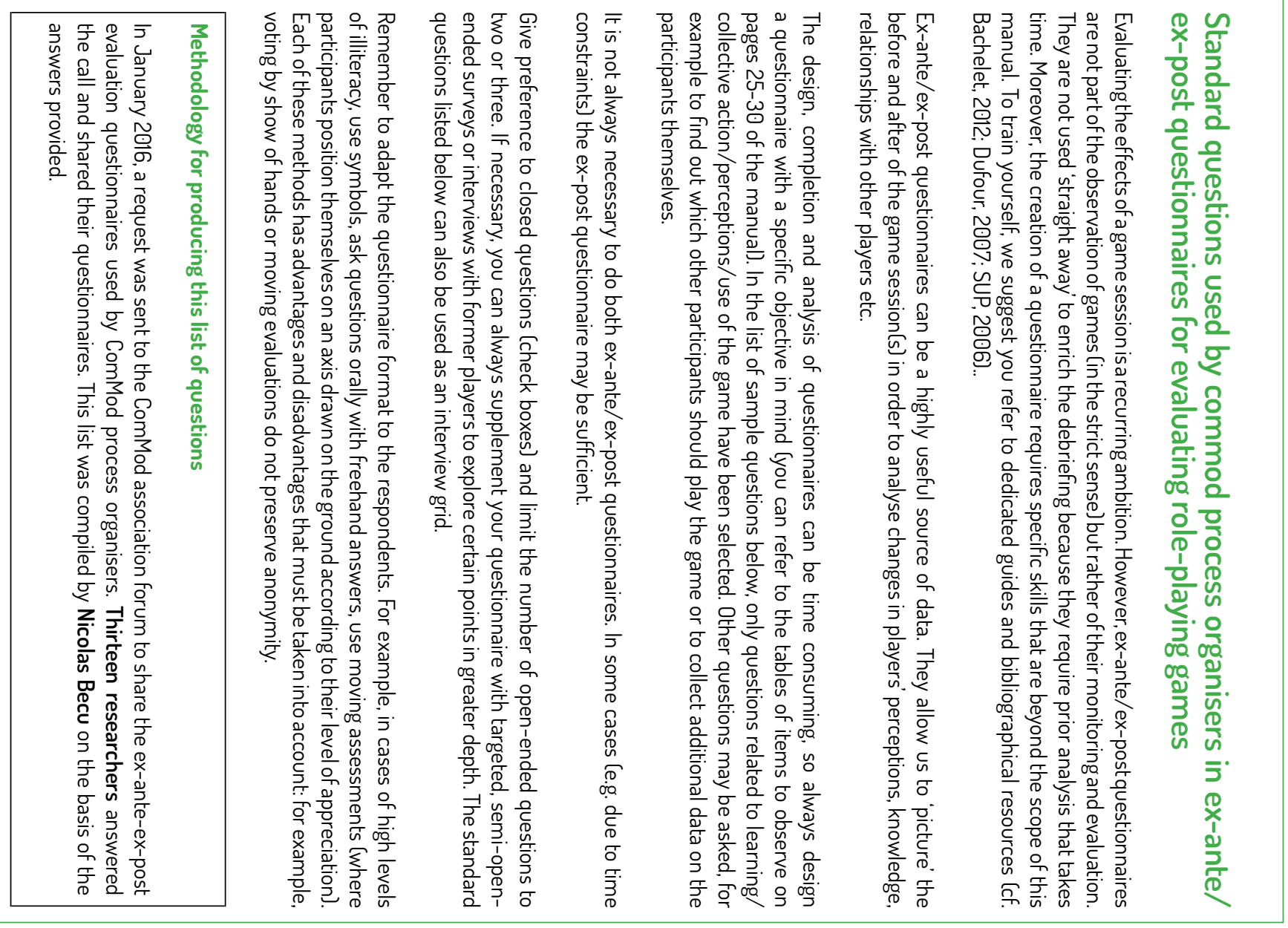




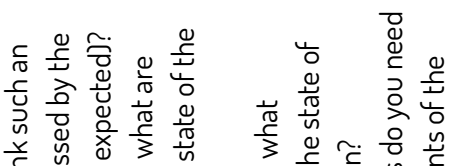

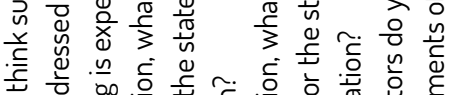


웡 उ

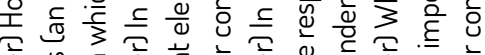

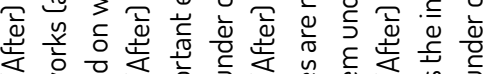

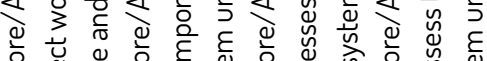
음 ญ 包

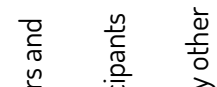

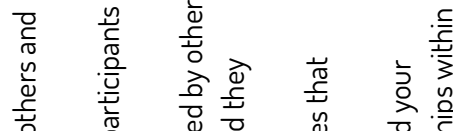

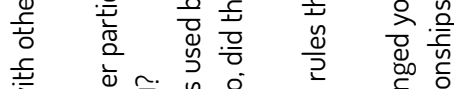

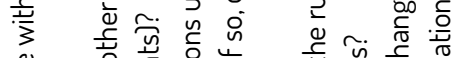

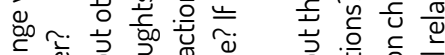
它 崩

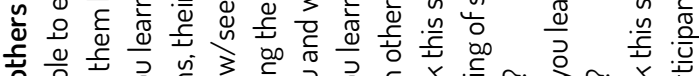

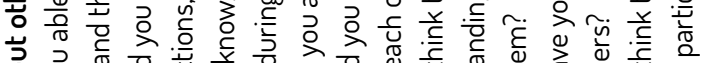

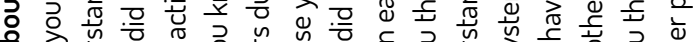

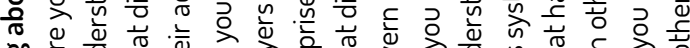
.

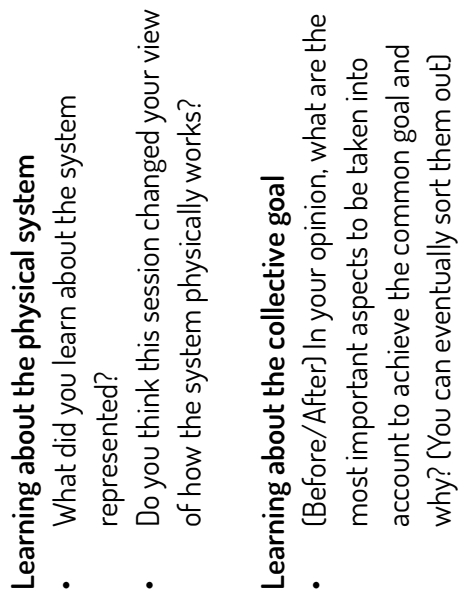

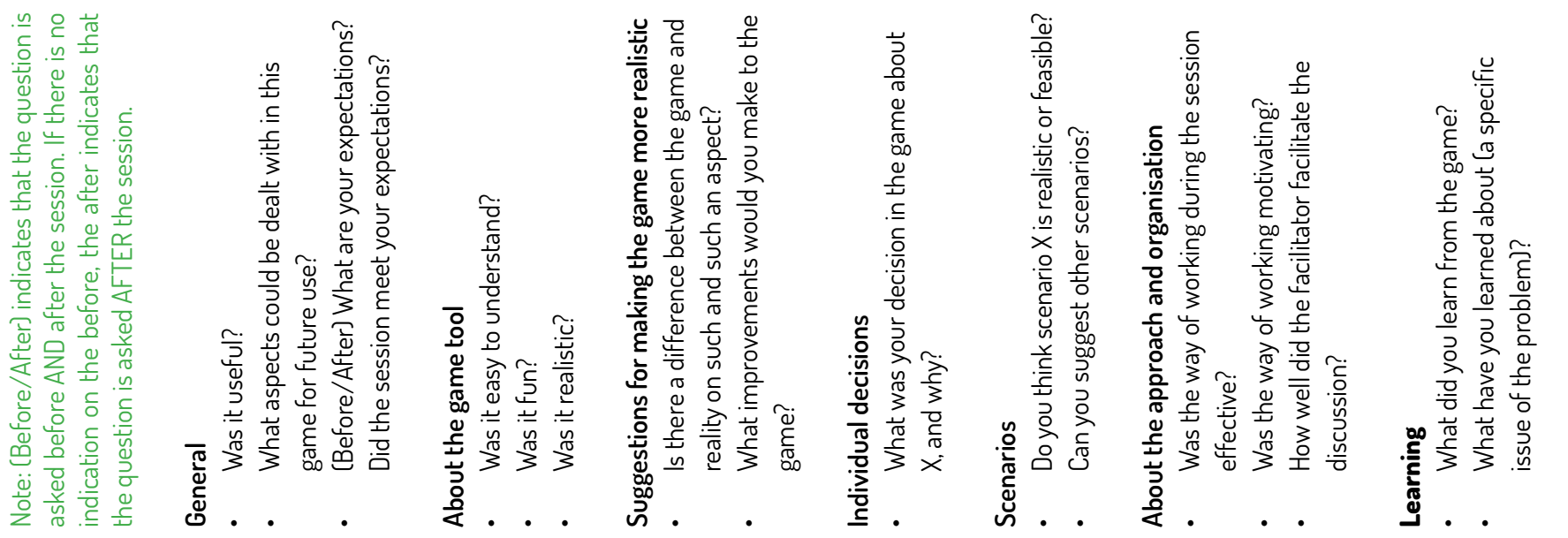




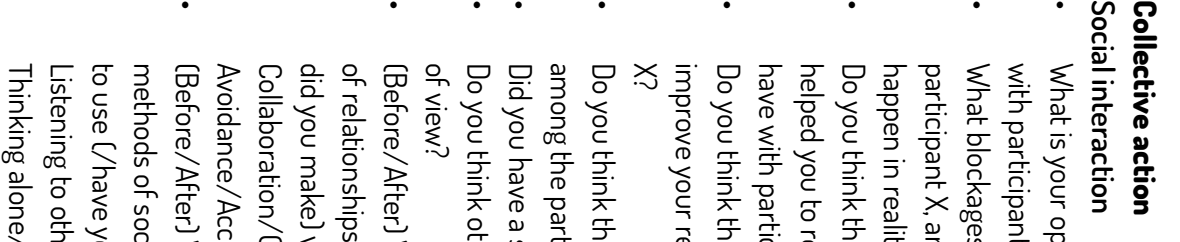
齐容 产. 虬

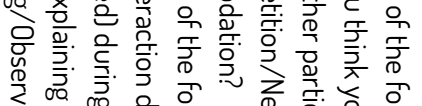

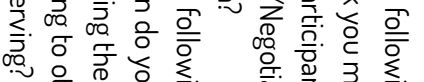

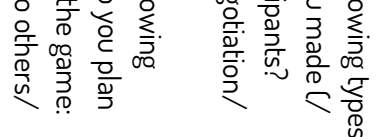

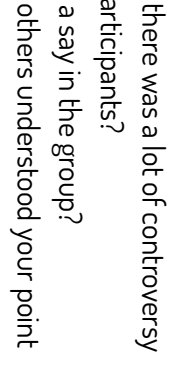

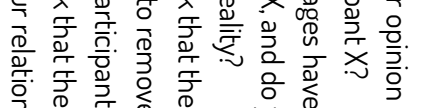

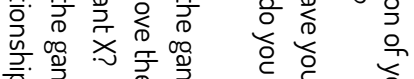

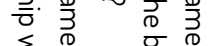
$\sum \frac{\pi}{\partial}$ 믕 $\frac{10}{3}$

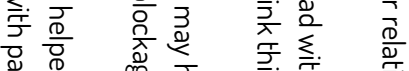

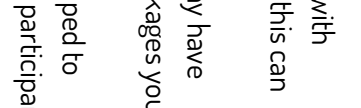

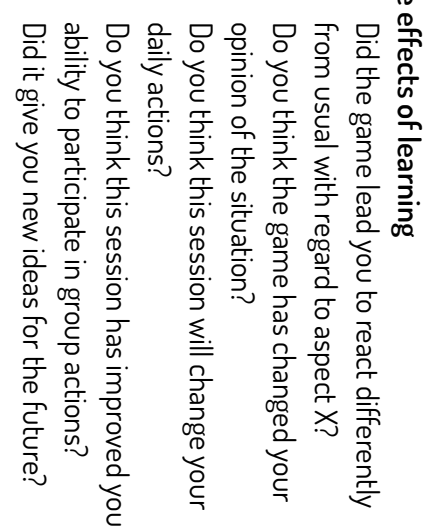

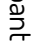

$\frac{\stackrel{2}{\frac{0}{0}}}{\stackrel{0}{\frac{0}{0}}}$

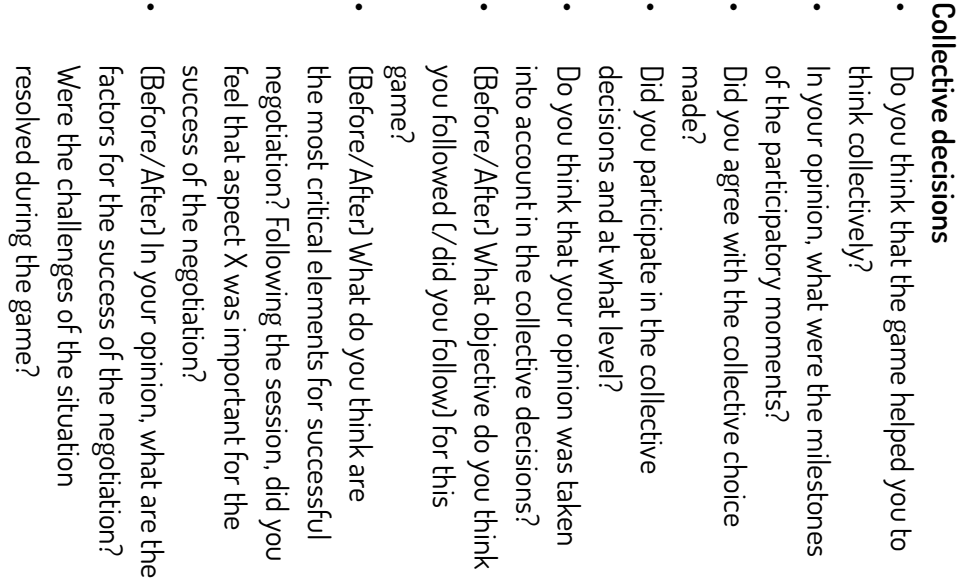




\section{ComMod Association}

A group of researchers and professors from CIRAD, CNRS, INRAE, IRD and French and international universities has developed and formalised an original way of modelling to support collective decision-making processes regarding the sustainable management of renewable natural resources." teacher-researchers from CIRAD, CNRS, INRAE, IRD and French and international universities has developed and formalised an original way of approaching modelling in support of collective decision-making processes regarding the sustainable management of renewable natural resources. This group, named ComMod (Companion Modelling), has become an association of practitioners.
The main purpose of ComMod is to design, analyse, develop and promote scientific research and its application in the field of Companion Modelling, including its main methods and tools, i.e. role-playing games, multi-agent modelling and social simulation.

The companion process aims to help different stakeholders gradually get to know each other, and to share their arguments and points of view in order to build a shared vision of a problem and to construct together an accepted solution. 


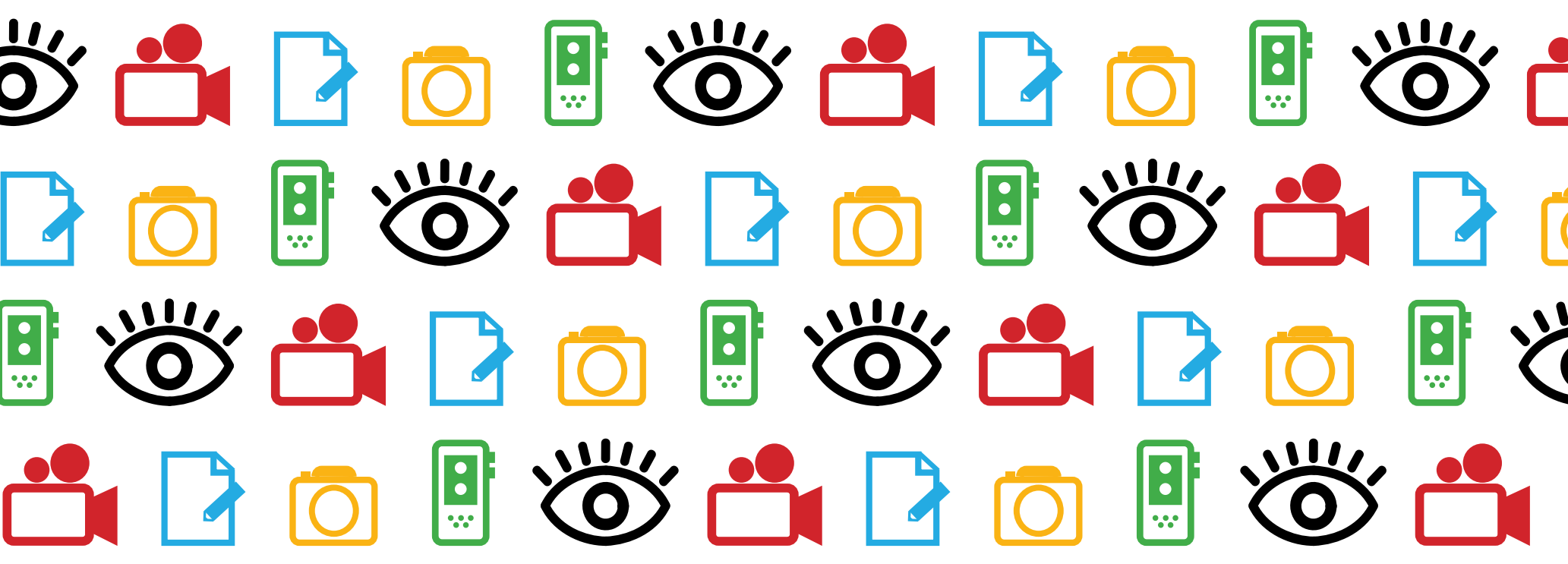

ComMod Association

11 Place Adolphe Chérioux

75015 Paris

France

www.commod.org

CIRAD

Avenue Agropolis

34398 Montpellier Cedex 5

France

www.cirad.fr

\section{cirad}

Comprod

Companion Modeling

EHHzürich

INRAC
ISBN : 978-2-87614-765-2

ComMod/CIRAD 2020 